\title{
BORCHERDS LIFTS OF HARMONIC MAASS FORMS AND MODULAR INTEGRALS
}

\author{
MARKUS SCHWAGENSCHEIDT
}

\begin{abstract}
We extend Borcherds' singular theta lift in signature $(1,2)$ to harmonic Maass forms of weight $1 / 2$ whose non-holomorphic part is allowed to be of exponential growth at $i \infty$. We determine the singularities of the lift and compute its Fourier expansion. It turns out that the lift is continuous but not differentiable along certain geodesics in the upper halfplane corresponding to the non-holomorphic principal part of the input. As an application, we obtain a generalization to higher level of the weight 2 modular integral of Duke, Imamoglu and Tóth. Further, we construct automorphic products associated to harmonic Maass forms.
\end{abstract}

\section{INTRODUCTION}

In [2, Borcherds constructed a regularized theta lift which maps weakly holomorphic modular forms of weight $1 / 2$ to real analytic modular functions with logarithmic singularities at CM points. His results were generalized by Bruinier and Ono [8] to twisted lifts of harmonic Maass forms which map to cusp forms under the $\xi$-operator. In the present work, we extend the twisted Borcherds lift to general harmonic Maass forms (which may map to weakly holomorphic modular forms under the $\xi$-operator). By taking the derivative of the Borcherds lift of a suitable harmonic Maass form of weight $1 / 2$, we obtain modular integrals of weight 2 with rational period functions. Their Fourier coefficients are given by twisted traces of geodesic cycle integrals of harmonic Maass forms of weight 0 . They generalize the modular integral of Duke, Imamoglu and Tóth [10] to higher level. In the introduction, we restrict to modular forms for the full modular group $\Gamma=\mathrm{SL}_{2}(\mathbb{Z})$ for simplicity, but in the body of the work we treat modular forms of arbitrary level $\Gamma_{0}(N)$ by using the language of vector valued modular forms for the Weil representation. Let us now describe our results in more detail.

1.1. The Borcherds lift of a harmonic Maass form. Recall from [5] that a harmonic Maass form of weight $1 / 2$ for $\Gamma_{0}(4)$ is a smooth function $f: \mathbb{H} \rightarrow \mathbb{C}$ which is annihilated by the invariant Laplace operator $\Delta_{1 / 2}$, transforms like a modular form of weight $1 / 2$ for $\Gamma_{0}(4)$, and is at most of linear exponential growth at the cusps of $\Gamma_{0}(4)$. Such a form can be written as a sum $f=f^{+}+f^{-}$with a holomorphic part $f^{+}$and a non-holomorphic part $f^{-}$with

Date: August 8, 2018.

This work is a shortened version of a chapter of my PhD thesis. I am indebted to my advisor Jan Bruinier for proposing the topic of this work to me and for many enlightening discussions. I also thank Kathrin Bringmann, Stephan Ehlen, and Yingkun Li for several helpful discussions on the topic. During the preparation of this work, I was partially supported by the DFG Research Unit FOR 1920 'Symmetry, Geometry and Arithmetic', by the LOEWE Reseach Unit USAG, and by the SFB-TRR 191 'Symplectic Structures in Geometry, Algebra and Dynamics', funded by the DFG . 
Fourier expansions of the shape

$$
\begin{aligned}
& f^{+}(\tau)=\sum_{D \in \mathbb{Z}} c_{f}^{+}(D) e(D \tau), \\
& f^{-}(\tau)=c_{f}^{-}(0) \sqrt{v}+\sum_{D<0} c_{f}^{-}(D) \sqrt{v} \beta_{1 / 2}(4 \pi|D| v) e(D \tau)+\sum_{D>0} c_{f}^{-}(D) \sqrt{v} \beta_{1 / 2}^{c}(-4 \pi D v) e(D \tau),
\end{aligned}
$$

with $\tau=u+i v \in \mathbb{H}, e(x)=e^{2 \pi i x}$ for $x \in \mathbb{C}$, coefficients $c_{f}^{ \pm}(D) \in \mathbb{C}$, and

$$
\beta_{1 / 2}(s)=\int_{1}^{\infty} e^{-s t} t^{-1 / 2} d t, \quad \beta_{1 / 2}^{c}(s)=\int_{0}^{1} e^{-s t} t^{-1 / 2} d t .
$$

We let $H_{1 / 2}$ denote the space of harmonic Maass forms of weight $1 / 2$ which satisfy the Kohnen plus space condition, which means that the Fourier expansion is supported on indices $D \equiv 0,1(4)$. The antilinear differential operator

$$
\xi_{1 / 2} f(\tau)=2 i v^{1 / 2} \overline{\frac{\partial}{\partial \bar{\tau}} f(\tau)}
$$

maps a harmonic Maass form $f \in H_{1 / 2}$ of weight $1 / 2$ to a weakly holomorphic modular form of weight $3 / 2$. We let $H_{1 / 2}^{+}$be the subspace of $H_{1 / 2}$ consisting of forms which map to cusp forms under $\xi_{1 / 2}$, and we let $M_{1 / 2}^{!}$be the subspace of weakly holomorphic modular forms.

Let $\Delta \in \mathbb{Z}$ be a fundamental discriminant. For simplicity, we assume $\Delta>1$ in the introduction. Following [2], we define the Borcherds lift $\Phi_{\Delta}(z, f)$ of a harmonic Maass form $f \in H_{1 / 2}$ by the regularized integral

$$
\Phi_{\Delta}(f, z)=\mathrm{CT}_{s=0}\left[\lim _{T \rightarrow \infty} \int_{\mathcal{F}_{T}(4)} f(\tau) \overline{\Theta_{\Delta}(\tau, z)} v^{1 / 2-s} \frac{d u d v}{v^{2}}\right],
$$

where $\Theta_{\Delta}(\tau, z)$ is a twisted Siegel theta function which transforms in $\tau$ like a modular form of weight $1 / 2$ for $\Gamma_{0}(4)$ and is invariant in $z$ under $\Gamma, \mathcal{F}_{T}(4)$ denotes a suitably truncated fundamental domain for $\Gamma_{0}(4) \backslash \mathbb{H}$, and $\mathrm{CT}_{s=0} F(s)$ denotes the constant term in the Laurent expansion at $s=0$ of a function $F(s)$ which is meromorphic near $s=0$. Borcherds [3] proved that for $\Delta=1$ and a weakly holomorphic modular form $f \in M_{1 / 2}^{!}$the regularized theta lift $\Phi_{\Delta}(f, z)$ defines a $\Gamma$-invariant real analytic function with logarithmic singularities at certain CM points in $\mathbb{H}$, which are determined by the principal part of $f$, i.e., by the coefficients $c_{f}^{+}(D)$ with $D<0$. Bruinier and Ono [8] showed that this result remains true for twisted Borcherds lifts of harmonic Maass forms $f \in H_{1 / 2}$ which map to cusp forms under the $\xi$-operator, which means that $c_{f}^{-}(0)=0$ and $c_{f}^{-}(D)=0$ for $D>0$. One of the main aims of the present work is to generalize the Borcherds lift $\Phi_{\Delta}(z, f)$ to the full space $H_{1 / 2}$.

For a discriminant $D$ we let let $\mathcal{Q}_{D}$ be the set of integral binary quadratic forms $Q=[a, b, c]$ of discriminant $D=b^{2}-4 a c$. For $D<0$ and $Q \in \mathcal{Q}_{D}$ there is an associated CM (or Heegner) point $z_{Q} \in \mathbb{H}$ which is characterized by $Q\left(z_{Q}, 1\right)=0$. For $D>0$ there is an associated geodesic in $\mathbb{H}$ given by

$$
c_{Q}=\left\{z \in \mathbb{H}: a|z|^{2}+b x+c=0\right\},
$$

with $z=x+i y \in \mathbb{H}$. We let $H_{\Delta}^{+}(f)$ be the set of all CM points $z_{Q}$ corresponding to quadratic forms $Q \in \mathcal{Q}_{\Delta D}$ with $D<0$ such that $c_{f}^{+}(D) \neq 0$, and we let $H_{\Delta}^{-}(f)$ be the union of all geodesics $c_{Q}$ corresponding to quadratic forms $Q \in \mathcal{Q}_{\Delta D}$ with $D>0$ such that $c_{f}^{-}(D) \neq 0$. We obtain the following extension of the Borcherds lift on the full space $H_{1 / 2}$. 
Theorem 1.1. Let $\Delta>1$ be a fundamental discriminant. For $f \in H_{1 / 2}$ the Borcherds lift $\Phi_{\Delta}(f, z)$ defines a $\Gamma$-invariant harmonic function on $\mathbb{H} \backslash\left(H_{\Delta}^{+}(f) \cup H_{\Delta}^{-}(f)\right)$. It has 'logarithmic singularities' at the $C M$ points in $H_{\Delta}^{+}(f)$ and 'arcsin singularities' along the geodesics in $H_{\Delta}^{-}(f)$. More precisely, this means that for $z_{0} \in H_{\Delta}^{+}(f) \cup H_{\Delta}^{-}(f)$ the function

$$
\begin{aligned}
\Phi_{\Delta}(f, z) & -\sum_{D<0} c_{f}^{+}(D) \sum_{\substack{Q=[a, b, c] \in \mathcal{Q}_{\Delta D} \\
z_{0}=z_{Q}}} \chi_{\Delta}(Q) \log \left|a z^{2}+b z+c\right| \\
& +\sum_{D>0} \frac{c_{f}^{-}(D)}{\sqrt{D}} \sum_{\substack{Q=[a, b, c] \in \mathcal{Q}_{\Delta D} \\
z_{0} \in c_{Q}}} \chi_{\Delta}(Q) \arcsin \left(\frac{1}{\sqrt{1+\frac{1}{\Delta D y^{2}}\left(a|z|^{2}+b x+c\right)^{2}}}\right) .
\end{aligned}
$$

can be continued to a real analytic function near $z_{0}$. Here $\chi_{\Delta}$ is the usual genus character. Note that all the above sums are finite.

We refer the reader to Theorem 3.1 for the general result.

Remark 1.2. The logarithmic singularities imply that the Borcherds lift blows up at the Heegner points $z_{Q} \in H_{\Delta}^{+}(f)$, and the arcsin singularities show that it is continuous but not differentiable at points on the geodesics $c_{Q} \subset H_{\Delta}^{-}(f)$.

1.2. The Fourier expansion of the Borcherds lift. Using Maass-Poincaré series one can always write a harmonic Maass form $f \in H_{1 / 2}$ as $f=f_{1}+f_{2}$ where $f_{1}, f_{2} \in H_{1 / 2}$ satisfy $c_{f_{1}}^{+}(D)=0$ for all $D<0$ and $c_{f_{2}}^{-}(D)=0$ for all $D \geq 0$. In particular, $f_{2}$ maps to a cusp form under the $\xi$-operator, and since the Borcherds lift of such harmonic Maass forms has already been investigated by Bruinier and Ono [8], we assume from now on that $c_{f}^{+}(D)=0$ for all $D<0$. In this case, the Borcherds lift $\Phi_{\Delta}(f, z)$ only has singularities along the geodesics in $H_{\Delta}^{-}(f)$. Furthermore, the Fourier expansion of $\Phi_{\Delta}(f, z)$ can be stated as follows.

Proposition 1.3. Let $\Delta>1$ be a fundamental discriminant and let $f \in H_{1 / 2}$ such that $c_{f}^{+}(D)=0$ for all $D<0$. Then for $z \in \mathbb{H} \backslash H_{\Delta}^{-}(f)$ the Borcherds lift of $f$ has the Fourier expansion

$$
\begin{aligned}
\Phi_{\Delta}(f, z)= & -4 \sum_{m=1}^{\infty} c_{f}^{+}\left(\Delta m^{2}\right) \sum_{b(\Delta)}\left(\frac{\Delta}{b}\right) \log |1-e(m z+b / \Delta)| \\
& +\sqrt{\Delta} L_{\Delta}(1)\left(2 c_{f}^{+}(0)+y c_{f}^{-}(0)\right) \\
& -4 \sum_{D>0} \frac{c_{f}^{-}(D)}{\sqrt{D}} \sum_{\substack{Q \in \mathcal{Q}_{\Delta D} \\
a>0}} \chi_{\Delta}(Q) \mathbf{1}_{Q}(z)\left(\arctan \left(\frac{y \sqrt{\Delta D}}{a|z|^{2}+b x+c}\right)+\frac{\pi}{2}\right),
\end{aligned}
$$

where $\mathbf{1}_{Q}(z)$ denotes the characteristic function of the bounded component of $\mathbb{H} \backslash c_{Q}$, and $L_{\Delta}(s)=\sum_{n \geq 1}\left(\frac{\Delta}{n}\right) n^{-s}$ for $\operatorname{Re}(s)>1$ is a Dirichlet L-function.

For the general result, see Proposition 4.2 .

Remark 1.4. (1) For $Q \in \mathcal{Q}_{\Delta D}$ with $a>0$ the corresponding geodesic $c_{Q}$ is a semi-circle centered at the real line which divides $\mathbb{H}$ into a bounded and an unbounded connected component, so the characteristic function $\mathbf{1}_{Q}$ makes sense. 
(2) The sum over $D$ in the third line is finite since $f$ has a finite principal part. The sum over $Q \in \mathcal{Q}_{\Delta D}$ is locally finite since each point $z \in \mathbb{H}$ lies in the bounded component of $\mathbb{H} \backslash c_{Q}$ for finitely many geodesics $c_{Q} \in \mathcal{Q}_{\Delta D}$, and it vanishes for $y \gg 0$ large enough since the imaginary parts of points lying on geodesics $c_{Q}$ for $Q \in \mathcal{Q}_{\Delta D}$ are bounded by $\sqrt{\Delta D}$.

(3) We have $z \in c_{Q}$ for $Q=[a, b, c]$ if and only if $a|z|^{2}+b x+c=0$. Further, for $a>0$ a point $z \in \mathbb{H}$ lies in the inside of the bounded component of $\mathbb{H} \backslash c_{Q}$ if and only if $a|z|^{2}+b x+c<0$. Since $\lim _{x \rightarrow-\infty} \arctan (x)=-\frac{\pi}{2}$, we see from the Fourier expansion that $\Phi_{\Delta}(f, z)$ is continuous. However, computing the derivative of the above expansion for $z \in \mathbb{H} \backslash H_{\Delta}^{-}(f)$ shows that the third line is not differentiable at points $z \in H_{\Delta}^{-}(f)$. More precisely, the derivative of $\Phi_{\Delta}(f, z)$ has jumps along the geodesics in $H_{\Delta}^{-}(f)$.

1.3. The derivative of the Borcherds lift. We apply the (derivative of the) Borcherds lift to certain interesting harmonic Maass forms of weight $1 / 2$ for $\Gamma_{0}(4)$, in order to construct modular integrals of weight 2 with rational period functions. In [10, Duke, Imamoglu and Tóth constructed a basis $\left\{h_{d}\right\}$ (indexed by discriminants $d>0$ ) of $H_{1 / 2}$, which under $\xi_{1 / 2}$ maps to a basis $\left\{g_{d}\right\}$ of the space of weakly holomorphic modular forms of weight $3 / 2$ for $\Gamma_{0}(4)$. More precisely, the $g_{d}$ are the generating series of traces of singular moduli, see [16]. The coefficients of the $h_{d}$ are given by traces of CM values and traces of (regularized) cycle integrals of weakly holomorphic modular functions for $\Gamma$. For example, the Fourier expansion of the function $h=h_{1}$ is given by

$$
h(\tau)=\frac{1}{2 \pi} \sum_{D>0} \operatorname{tr}_{J}(D) q^{D}+2 \sqrt{v} \beta_{1 / 2}^{c}(-4 \pi v) q-8 \sqrt{v}+\sqrt{v} \sum_{D<0} \operatorname{tr}_{J}(D) \beta_{1 / 2}(4 \pi|D| v) q^{D}
$$

where

$$
\operatorname{tr}_{J}(D)= \begin{cases}\sum_{Q \in \mathcal{Q}_{D} / \Gamma} \frac{J\left(z_{Q}\right)}{\left|\bar{\Gamma}_{Q}\right|}, & D<0, \\ \sum_{Q \in \mathcal{Q}_{D} / \Gamma} \int_{\Gamma_{Q} \backslash c_{Q}} J(z) \frac{d z}{Q(z, 1)}, & D>0,\end{cases}
$$

are traces of $\mathrm{CM}$ values and geodesic cycle integrals of $J=j-744$, which need to be regularized as explained in [7] if $D>0$ is a square. The harmonic Maass form $h$ does not map to a cusp form but to a weakly holomorphic modular form under $\xi_{1 / 2}$, so it is interesting to apply our extension of the Borcherds lift to it. The coefficients $c_{h}^{+}(D)$ for $D \leq 0$ vanish, so the Borcherds lift $\Phi_{\Delta}(h, z)$ is a harmonic $\Gamma$-invariant function on $\mathbb{H} \backslash H_{\Delta}^{-}(h)$ with arcsin singularities along the geodesics in $H_{\Delta}^{-}(h)$. In this case, the latter set is just the union of all geodesics $c_{Q}$ for $Q \in \mathcal{Q}_{\Delta}$. Hence the derivative $\Phi_{\Delta}^{\prime}(h, z)=\frac{\partial}{\partial z} \Phi_{\Delta}(h, z)$ is a holomorphic function on $\mathbb{H} \backslash H_{\Delta}^{-}(h)$ transforming like a modular form of weight 2 for $\Gamma$. Moreover, it turns out that $\Phi_{\Delta}^{\prime}(h, z)$ has jump singularities along the geodesics in $H_{\Delta}^{-}(h)$, and admits a nice Fourier expansion.

Proposition 1.5. Let $\Delta>1$ be a fundamental discriminant. The derivative $\Phi_{\Delta}^{\prime}(h, z)$ of the Borcherds lift of $h$ is a holomorphic function on $\mathbb{H} \backslash H_{\Delta}^{-}(h)$ which transforms like a modular 
form of weight 2 for $\Gamma$. For $z \in \mathbb{H} \backslash H_{\Delta}^{-}(h)$ it has the expansion

$$
\begin{aligned}
& \frac{1}{4 \pi i \sqrt{\Delta}} \Phi_{\Delta}^{\prime}(h, z) \\
& =\frac{1}{2 \pi} \operatorname{tr}_{1}(\Delta)+\frac{1}{2 \pi} \sum_{n=1}^{\infty}\left(\sum_{m \mid n}\left(\frac{\Delta}{n / m}\right) m \operatorname{tr}_{J}\left(\Delta m^{2}\right)\right) e(n z)+\frac{1}{\pi} \sum_{\substack{Q \in \mathcal{Q}_{\Delta} \\
a>0}} \frac{\mathbf{1}_{Q}(z)}{Q(z, 1)},
\end{aligned}
$$

where $\mathbf{1}_{Q}$ denotes the characteristic function of the bounded component of $\mathbb{H} \backslash c_{Q}$.

The result for general harmonic Maass forms $f \in H_{1 / 2}$ of higher level is given in Proposition 5.3 and Corollary 5.4 ,

Remark 1.6. The Fourier series over $n$ is holomorphic on $\mathbb{H}$, whereas the sum over $Q$ has jump singularities along the geodesics $c_{Q}$ with $Q \in \mathcal{Q}_{\Delta}$. Again, the sum over $Q$ is locally finite and vanishes for $y \gg 0$ large enough.

1.4. Modular integrals. In [10], Theorem 5, the authors proved that the generating series

$$
F_{\Delta}(z)=\frac{1}{\pi} \sum_{m=0}^{\infty} \operatorname{tr}_{J_{m}}(\Delta) e(m z),
$$

with $J_{m}(z)=q^{-m}+O(q) \in M_{0}^{!}$, e.g. $J_{0}=1$ and $J_{1}=J$, defines a holomorphic function on $\mathbb{H}$ which transforms as

$$
z^{-2} F_{\Delta}\left(-\frac{1}{z}\right)-F_{\Delta}(z)=\frac{2}{\pi} \sum_{\substack{Q \in \mathcal{Q}_{\Delta} \\ c<0<a}} \frac{1}{Q(z, 1)},
$$

so $F_{\Delta}(z)$ is a holomorphic modular integral of weight 2 with holomorphic rational period functions in the sense of [14].

Returning to the derivative of the Borcherds lift of $h$, we note that

$$
\operatorname{tr}_{J_{m}}(\Delta)=\sum_{d \mid m}\left(\frac{\Delta}{m / d}\right) d \operatorname{tr}_{J_{1}}\left(\Delta d^{2}\right),
$$

compare [15], pp. 290-292, so $F_{\Delta}(z)$ in fact agrees with $\Phi_{\Delta}^{\prime}(h, z)$ up to some constant factor if $y \gg 0$ is sufficiently large. The transformation behaviour of the singular part in the Fourier expansion of $\Phi_{\Delta}^{\prime}(h, z)$ can easily be determined, so we can recover (11) from Proposition 1.5. Further, using the Borcherds lift we generalize the construction of modular integrals of weight 2 with rational period functions from [10] to higher level, see Proposition 6.1. The coefficients of our modular integrals are linear combinations of Fourier coefficients of the holomorphic parts of harmonic Maass forms $f$ of weight $1 / 2$. Choosing $f$ as the image of a theta lift of a harmonic Maass form $F$ of weight 0 studied by Bruinier, Funke and Imamoglu [7, we obtain modular integrals whose coefficients are linear combinations of traces of cycle integrals of $F$, see Example 6.3. In fact, the construction of $F_{\Delta}$ as a theta lift and its generalizations to higher level were our main motivation to extend the Borcherds lift to the full space $H_{1 / 2}$.

1.5. Borcherds products. Bruinier and Ono [8] defined a twisted Borcherds product associated to a harmonic Maass form $f \in H_{1 / 2}^{+}$with real coefficients $c_{f}^{+}(D)$ for all $D$, and 
$c_{f}^{+}(D) \in \mathbb{Z}$ for $D \leq 0$. For $\Delta>1$ a fundamental discriminant and $y \gg 0$ sufficiently large the twisted Borcherds lift of $f$ is given by

$$
\Psi_{\Delta}(f, z)=\prod_{m=1}^{\infty} \prod_{b(\Delta)}[1-e(m z+b / \Delta)]^{\left(\frac{\Delta}{b}\right) c_{f}^{+}\left(\Delta m^{2}\right)} .
$$

It has a meromorphic continuation to $\mathbb{H}$ with roots and poles at $\mathrm{CM}$ points corresponding to the principal part of $f$, and it transforms like a modular form of weight 0 with some unitary character for $\Gamma$. We will define Borcherds products associated to general harmonic Maass forms $f \in H_{1 / 2}$. For simplicity, in the introduction we only consider the harmonic Maass form $\pi h$. The general result is given in Theorem 6.8.

Theorem 1.7. Let $\Delta>1$ be a fundamental discriminant. Then the infinite product

$$
\Psi_{\Delta}(z)=e\left(-\sqrt{\Delta} \operatorname{tr}_{1}(\Delta) z\right) \prod_{m=1}^{\infty} \prod_{b(\Delta)}[1-e(m z+b / \Delta)]^{\left(\frac{\Delta}{b}\right) \operatorname{tr}_{J}\left(\Delta m^{2}\right)}
$$

converges to a holomorphic function on $\mathbb{H}$. Its logarithmic derivative is given by

$$
\frac{\partial}{\partial z} \log \left(\Psi_{\Delta}(z)\right)=-2 \pi^{2} i \sqrt{\Delta} F_{\Delta}(z)
$$

Further, it transforms as

$$
\begin{aligned}
& \Psi_{\Delta}(z+1)=e\left(-\sqrt{\Delta} \operatorname{tr}_{1}(\Delta)\right) \Psi_{\Delta}(z) \\
& \Psi_{\Delta}\left(-\frac{1}{z}\right)=e\left(-2 \sum_{\substack{Q \in \mathcal{Q}_{\Delta} \\
c<0<a}}\left(\log \left(\frac{z-w_{Q}}{i-w_{Q}}\right)-\log \left(\frac{z-w_{Q}^{\prime}}{i-w_{Q}^{\prime}}\right)\right)\right) \Psi_{\Delta}(z),
\end{aligned}
$$

where $w_{Q}>w_{Q}^{\prime}$ denote the real endpoints of the geodesic $c_{Q}$.

The work is organized as follows. We start with a section on the necessary preliminaries about the Grassmannian model of the upper half-plane, which is convenient for the study of regularized theta lifts, and vector valued harmonic Maass forms for the Weil representation.

In Section 3, we define the Borcherds lift of a harmonic Maass form of weight $1 / 2$ and prove its basic analytic properties.

In Section 4 we compute the Fourier expansion of the Borcherds lift. After that, in Section 5 we compute the Fourier expansion of the derivative of the Borcherds lift and show that it has jump singularities along geodesics.

In Section 6.1 we apply the derivative of the Borcherds lift to an interesting class of harmonic Maass forms which arise as images of regularized theta lifts of scalar valued weight 0 harmonic Maass forms $F$. We show that the generating series of certain sums of traces of cycle integrals of $F$ transform like modular forms of weight 2 for $\Gamma_{0}(N)$ and are holomorphic up to jump singularities along geodesics. Their non-singular parts yield holomorphic modular integrals of weight 2 for $\Gamma_{0}(N)$ with rational period functions.

Finally, in Section 6.2 we define the Borcherds product associated to a general harmonic Maass form of weight $1 / 2$ and prove its modularity. 


\section{Preliminaries}

2.1. The Grassmannian model of the upper half-plane. For a positive integer $N$ we consider the quadratic space $V$ of all rational traceless 2 by 2 matrices, equipped with the quadratic form $Q(X)=N \operatorname{det}(X)$ and the associated bilinear form $(X, Y)=-N \operatorname{tr}(X Y)$. It has signature $(1,2)$. We let $D$ be the Grassmannian of positive definite lines in $V(\mathbb{R})=V \otimes \mathbb{R}$. We identify it with the complex upper half-plane $\mathbb{H}$ by associating to $z=x+i y \in \mathbb{H}$ the line spanned by

$$
X(z)=\frac{1}{\sqrt{2 N} y}\left(\begin{array}{cc}
-x & |z|^{2} \\
-1 & x
\end{array}\right)
$$

The group $\mathrm{SL}_{2}(\mathbb{R})$ acts as isometries on $V(\mathbb{R})$ by conjugation and this action is compatible with the action by fractional linear transformations on $\mathbb{H}$ under the above identification.

For $X \in V$ and $z \in D$ we let $X_{z}$ and $X_{z^{\perp}}$ denote the projection of $X$ to $z$ and its orthogonal complement $z^{\perp}$, respectively.

2.2. A lattice related to $\Gamma_{0}(N)$. In $V$ we consider the even lattice

$$
L=\left\{\left(\begin{array}{cc}
-b & -c / N \\
a & b
\end{array}\right): a, b, c \in \mathbb{Z}\right\}
$$

Its dual lattice is given by

$$
L^{\prime}=\left\{\left(\begin{array}{cc}
-b / 2 N & -c / N \\
a & b / 2 N
\end{array}\right): a, b, c \in \mathbb{Z}\right\}
$$

We see that $L^{\prime} / L \cong \mathbb{Z} / 2 N \mathbb{Z}$, and we will use this identification without further notice in the following. For $m \in \mathbb{Q}$ and $h \in L^{\prime} / L$ we let

$$
L_{m, h}=\{X \in L+h: Q(X)=m\} .
$$

The group $\Gamma_{0}(N)$ acts on $L_{m, h}$, with finitely many orbits if $m \neq 0$.

Let $X \in L_{m, h}$. If $m>0$ we let

$$
z_{X}=\operatorname{span}(X) \in D
$$

be the associated CM (or Heegner) point. If $m<0$ we let

$$
c_{X}=\{z \in D: z \perp X\}
$$

be the associated geodesic in $D$. We use the same symbols for the corresponding points and geodesics in $\mathbb{H}$. We can identify $X=[a, b, c] \in L^{\prime}$ with the binary quadratic form $Q_{X}=[a N, b, c]$. Under this identification, the set $L_{m, h}$ corresponds to the set of all binary quadratic forms $[a N, b, c]$ with $a, b, c \in \mathbb{Z}$ of discriminant $-4 N m$ and $b \equiv h(2 N)$. Furthermore, CM points and geodesics associated to vectors $X \in L_{m, h}$ correspond to the usual CM points and geodesics associated to $Q_{X}$. We define the quantity

$$
p_{X}(z)=-\frac{a N|z|^{2}+b x+c}{y \sqrt{N}},
$$

which vanishes exactly at the geodesic $c_{X}$. 
2.3. Vector valued harmonic Maass forms for the Weil representation. Let $\tilde{\Gamma}=$ $\mathrm{Mp}_{2}(\mathbb{Z})$ be the integral metaplectic group, realized as the set of pairs $(M, \phi)$ with $M=$ $\left(\begin{array}{ll}a & b \\ c & d\end{array}\right) \in \mathrm{SL}_{2}(\mathbb{Z})$ and $\phi: \mathbb{H} \rightarrow \mathbb{C}$ holomorphic with $\phi^{2}(\tau)=c \tau+d$. We let $\tilde{\Gamma}_{\infty}$ be the subgroup of $\tilde{\Gamma}$ generated by $\tilde{T}=\left(\left(\begin{array}{ll}1 & 1 \\ 0 & 1\end{array}\right), 1\right)$. Let $\mathbb{C}\left[L^{\prime} / L\right]$ be the group ring of $L^{\prime} / L$, generated by the formal basis vector $\mathfrak{e}_{\gamma}$ for $\gamma \in L^{\prime} / L$. We let $\langle\cdot, \cdot\rangle$ be the inner product on $\mathbb{C}\left[L^{\prime} / L\right]$ which is antilinear in the second variable and satisfies $\left\langle\mathfrak{e}_{\gamma}, \mathfrak{e}_{\beta}\right\rangle=\delta_{\gamma, \beta}$. The Weil representation $\rho_{L}$ is a unitary representation of $\tilde{\Gamma}$ on $\mathbb{C}\left[L^{\prime} / L\right]$, see [3], Section 4 . We let $\rho_{L}^{*}$ be the dual Weil representation.

A harmonic Maass form of weight $1 / 2$ for $\rho_{L}$ is a harmonic function $f: \mathbb{H} \rightarrow \mathbb{C}\left[L^{\prime} / L\right]$ which transforms like a modular form of weight $1 / 2$ for $\rho_{L}$ and is at most of linear exponential growth at $i \infty$. We let $H_{1 / 2, \rho_{L}}$ be the space of all harmonic Maass forms of weight $1 / 2$ for $\rho_{L}$. Every $f \in H_{1 / 2, \rho_{L}}$ can be written as a sum $f=f^{+}+f^{-}$of a holomorphic and a non-holomorphic part having Fourier expansions of the form

$$
\begin{aligned}
f^{+}= & \sum_{h \in L^{\prime} / L} \sum_{n \gg-\infty} c_{f}^{+}(n, h) e(n \tau) \mathfrak{e}_{h}, \\
f^{-}= & \sum_{h \in L^{\prime} / L}\left(c_{f}^{-}(0, h) \sqrt{v}+\sum_{n<0} c_{f}^{-}(n, h) \beta_{1 / 2}(-4 \pi n v) e(n \tau)\right. \\
& \left.\quad+\sum_{0<n \ll \infty} c_{f}^{-}(n, h) \beta_{1 / 2}^{c}(-4 \pi n v) e(n \tau)\right) \mathfrak{e}_{h},
\end{aligned}
$$

with coefficients $c_{f}^{ \pm}(D) \in \mathbb{C}, e(x)=e^{2 \pi i x}$ for $x \in \mathbb{C}$, and

$$
\beta_{1 / 2}(s)=\int_{1}^{\infty} e^{-s t} t^{-1 / 2} d t, \quad \beta_{1 / 2}^{c}(s)=\int_{0}^{1} e^{-s t} t^{-1 / 2} d t .
$$

Remark 2.1. For $N=1$ the space $H_{1 / 2, \rho_{L}}$ of vector valued harmonic Maass forms is isomorphic to the space $H_{1 / 2}$ of scalar valued harmonic Maass forms from the introduction. The isomorphism is given by the map $f_{0}(\tau) \mathfrak{e}_{0}+f_{1}(\tau) \mathfrak{e}_{1} \mapsto f_{0}(4 \tau)+f_{1}(4 \tau)$, compare [12], Theorem 5.1. This identification can be used to translate the results from the body of the paper to the scalar valued setup in the introduction.

\section{Analytic properties of the Borcherds lift}

In this section we extend Borcherds' regularized theta lift to general harmonic Maass forms of weight $1 / 2$.

Let $\Delta \in \mathbb{Z}$ be a fundamental discriminant (possibly 1) and let $r \in \mathbb{Z} / 2 N \mathbb{Z}$ such that $\Delta \equiv r^{2} \bmod 4 N$. We set

$$
\tilde{\rho}_{L}=\left\{\begin{array}{ll}
\rho_{L}, & \text { if } \Delta>0, \\
\rho_{L}^{*}, & \text { if } \Delta<0,
\end{array} \quad Q_{\Delta}(X)=\frac{1}{|\Delta|} Q(X), \quad(X, Y)_{\Delta}=\frac{1}{|\Delta|}(X, Y) .\right.
$$

We consider the twisted Siegel theta function

$$
\Theta_{\Delta, r}(\tau, z)=v \sum_{h \in L^{\prime} / L} \sum_{\substack{X \in L+r h \\ Q(X) \equiv \Delta Q(h)(\Delta)}} \chi_{\Delta}(X) e\left(\tau Q_{\Delta}\left(X_{z}\right)+\bar{\tau} Q_{\Delta}\left(X_{z^{\perp}}\right)\right) \mathfrak{e}_{h},
$$

which is $\Gamma_{0}(N)$-invariant in $z$ and transforms like a modular form of weight $-1 / 2$ for $\tilde{\rho}_{L}$, compare [8], Theorem 4.1. Here $\chi_{\Delta}$ is the genus character defined as in [8], Section 4. 
For $f \in H_{1 / 2, \tilde{\rho}_{L}^{*}}$ we let

$$
H_{\Delta, r}^{+}(f)=\bigcup_{\substack{h \in L^{\prime} / L, n<0 \\ c_{f}^{+}(n, h) \neq 0}}\left\{z_{X}: X \in L_{-|\Delta| n, r h}\right\}, \quad H_{\Delta, r}^{-}(f)=\bigcup_{\substack{h \in L^{\prime} / L, n>0 \\ c_{f}^{-}(n, h) \neq 0}} \bigcup_{X \in L_{-|\Delta| n, r h}} c_{X},
$$

be the sets of Heegner points and geodesics associated to $f$.

Following Borcherds [3], we define the regularized theta lift of $f \in H_{1 / 2, \tilde{\rho}_{L}^{*}}$ by

$$
\Phi_{\Delta, r}(f, z)=\mathrm{CT}_{s=0}\left(\lim _{T \rightarrow \infty} \int_{\mathcal{F}_{T}}\left\langle f(\tau), \overline{\Theta_{\Delta, r}(\tau, z)}\right\rangle v^{-s} \frac{d u d v}{v^{2}}\right),
$$

where

$$
\mathcal{F}_{T}=\{\tau=u+i v \in \mathbb{H}:|\tau| \geq 1,|u| \leq 1 / 2, v \leq T\}
$$

is a truncated fundamental domain for the action of $\mathrm{SL}_{2}(\mathbb{Z})$ on $\mathbb{H}$, and $\mathrm{CT}_{s=0} F(s)$ denotes the constant term in the Laurent expansion of the analytic continuation of $F(s)$ at $s=0$.

We say that a complex-valued function $f$ defined on some subset of $\mathbb{R}^{n}$ has a singularity of type $g$ (written $f \approx g$ ) at a point $z_{0}$ if there is an open neighbourhood $U$ of $z_{0}$ such that $f$ and $g$ are defined on a dense subset of $U$ and $f-g$ can be continued to a real analytic function on $U$.

Theorem 3.1. For $f \in H_{1 / 2, \tilde{\rho}_{L}^{*}}$ the Borcherds lift $\Phi_{\Delta, r}(f, z)$ defines a $\Gamma_{0}(N)$-invariant real analytic function on $\mathbb{H} \backslash\left(H_{\Delta, r}^{+}(f) \cup H_{\Delta, r}^{-}(f)\right)$ with

$$
\Delta_{0} \Phi_{\Delta, r}(f, z)= \begin{cases}-2 c_{f}^{+}(0,0), & \text { if } \Delta=1, \\ 0, & \text { if } \Delta \neq 1 .\end{cases}
$$

At a point $z_{0} \in H_{\Delta, r}^{+}(f) \cup H_{\Delta, r}^{-}(f)$ it has a singularity of type

$$
\begin{aligned}
& -\sum_{h \in L^{\prime} / L} \sum_{n<0} c_{f}^{+}(n, h) \sum_{\substack{X \in L_{-|| \mid n, r h} \\
z_{0}=z_{X}}} \chi_{\Delta}(X) \log \left(-Q_{\Delta}\left(X_{z^{\perp}}\right)\right) \\
& +\sum_{h \in L^{\prime} / L} \sum_{n>0} c_{f}^{-}(n, h) n^{-1 / 2} \sum_{\substack{X \in L_{-}|\Delta| n, r h \\
z_{0} \in c_{X}}} \chi_{\Delta}(X) \arcsin \left(\sqrt{\frac{Q_{\Delta}(X)}{Q_{\Delta}\left(X_{z^{\perp}}\right)}}\right) .
\end{aligned}
$$

\section{Remark 3.2.}

(1) For $X=\left(\begin{array}{cc}-b / 2 N & -c / N \\ a & b / 2 N\end{array}\right) \in L^{\prime}$ we have

$$
-Q_{\Delta}\left(X_{z^{\perp}}\right)=\frac{1}{4 N|\Delta| y^{2}}\left|Q_{X}(z)\right|^{2}=\frac{1}{4 N|\Delta| y^{2}}\left|a N z^{2}+b z+c\right|^{2},
$$

which yields a more explicit formula for the singularities. Since $0<\frac{Q_{\Delta}(X)}{Q_{\Delta}\left(X_{z} \perp\right)} \leq 1$ for all $X$ with $Q_{\Delta}(X)<0$, and $Q_{\Delta}(X) / Q_{\Delta}\left(X_{z^{\perp}}\right)=1$ exactly for $z \in c_{X}$, we see that the Borcherds lift extends to a continuous function on $\mathbb{H} \backslash H_{\Delta, r}^{+}(f)$, which is not differentiable along the geodesics in $H_{\Delta, r}^{-}(f)$. Note that we can also write the singularities in the form

$$
\arcsin \left(\sqrt{\frac{Q_{\Delta}(X)}{Q_{\Delta}\left(X_{z^{\perp}}\right)}}\right)=\arctan \left(\sqrt{\frac{Q_{\Delta}(X)}{-Q_{\Delta}\left(X_{z}\right)}}\right) .
$$


(2) For every exact divisor $d \| N$ (i.e., $d \mid N$ and $(d, N / d)=1)$ the Atkin-Lehner involution $W_{d}$ acts on the Siegel theta function by

$$
\Theta_{\Delta, r}\left(\tau, W_{d} z\right)=\Theta_{\Delta, r}(\tau, z)^{w_{d}},
$$

where $w_{d}$ is the orthogonal map on $L^{\prime} / L$ defined by $w_{d}(h) \equiv-h(2 d)$ and $w_{d}(h) \equiv$ $h(2 N / d)$, which acts on $f=\sum_{h} f_{h} \mathfrak{e}_{h}$ by $f^{w_{d}}=\sum_{h} f_{h} \mathfrak{e}_{w_{d}(h)}$. This implies that the Borcherds lift satisfies

$$
\Phi_{\Delta, r}\left(f, W_{d} z\right)=\Phi_{\Delta, r}\left(f^{w_{d}}, z\right) .
$$

Proof of Theorem 3.1. We first show that for $z \in \mathbb{H} \backslash\left(H_{\Delta, r}^{+}(f) \cup H_{\Delta, r}^{-}(f)\right)$ the integral in (4) converges absolutely and locally uniformly for $\operatorname{Re}(s)>1 / 2$ and has a meromorphic continuation to $s=0$. The proof follows the arguments of [4], Proposition 2.8.

The integral over the compact set $\mathcal{F}_{1}=\{\tau \in \mathbb{H}:|\tau| \geq 1,|u| \leq 1 / 2, v \leq 1\}$ converges absolutely and locally uniformly for all $s \in \mathbb{C}$ and $z \in \mathbb{H}$. We consider the remaining integral

$$
\varphi(z, s)=\int_{v=1}^{\infty} \int_{u=0}^{1}\left\langle f(\tau), \overline{\Theta_{\Delta, r}(\tau, z)}\right\rangle v^{-s} \frac{d u d v}{v^{2}} .
$$

Inserting the Fourier expansions of $f(\tau)$ and $\Theta_{\Delta, r}(\tau, z)$ and carrying out the integral over $u$, we obtain

$$
\begin{aligned}
& \varphi(z, s)=\chi_{\Delta}(0)\left(c_{f}^{+}(0,0) \int_{v=1}^{\infty} v^{-1-s} d v+c_{f}^{-}(0,0) \int_{v=1}^{\infty} v^{-1 / 2-s} d v\right) \\
& +\int_{v=1}^{\infty} \sum_{h, X} \chi_{\Delta}(X) c_{f}^{+}\left(-Q_{\Delta}(X), h\right) \exp \left(4 \pi Q_{\Delta}\left(X_{z^{\perp}}\right) v\right) v^{-s-1} d v \\
& \quad+\int_{v=1}^{\infty} \sum_{Q(X)=0} \chi_{\Delta}(X) c_{f}^{-}\left(-Q_{\Delta}(X), h\right) \exp \left(4 \pi Q_{\Delta}\left(X_{z^{\perp}}\right) v\right) v^{-s-1 / 2} d v \\
& \quad+\int_{v=1}^{\infty} \sum_{Q(X)>0} \chi_{\Delta}(X) c_{f}^{-}\left(-Q_{\Delta}(X), h\right) \beta_{1 / 2}\left(4 \pi Q_{\Delta}(X) v\right) \exp \left(4 \pi Q_{\Delta}\left(X_{z^{\perp}}\right) v\right) v^{-s-1 / 2} d v \\
& \quad+\int_{v=1}^{\infty} \sum_{Q(X)<0} \chi_{\Delta}(X) c_{f}^{-}\left(-Q_{\Delta}(X), h\right) \beta_{1 / 2}^{c}\left(4 \pi Q_{\Delta}(X) v\right) \exp \left(4 \pi Q_{\Delta}\left(X_{z^{\perp}}\right) v\right) v^{-s-1 / 2} d v
\end{aligned}
$$

where the sums run over $h \in L^{\prime} / L$ and $X \in(L+r h) \backslash\{0\}$ with $Q(X) \equiv \Delta Q(h) \bmod \Delta$.

Since $\chi_{\Delta}(0)=0$ for $\Delta \neq 1$ the integrals in the first line only appear if $\Delta=1$. They can be evaluated for $\operatorname{Re}(s)>1 / 2$ by

$$
\int_{v=1}^{\infty} v^{-1-s} d v=\frac{1}{s}, \quad \int_{v=1}^{\infty} v^{-1 / 2-s} d v=\frac{1}{s-1 / 2}
$$

giving their meromorphic continuations to $s=0$. Note that this shows that for $\Delta=1$ the regularization involving the extra parameter $s$ is really necessary.

The integral in the second line involving the coefficients $c_{f}^{+}(n, h)$ converges locally uniformly and absolutely for $s \in \mathbb{C}$ and $z \in \mathbb{H} \backslash H_{\Delta, r}^{+}(f)$ by the same arguments as in the proof of [4], Proposition 2.8. The integrals over the sums corresponding to $Q(X)=0$ and $Q(X)>0$ in the third and fourth line can be treated in the same way, and they converge locally uniformly and absolutely for $s \in \mathbb{C}$ and $z \in \mathbb{H}$. 
The remaining integral in the fifth line can be written as

$$
\sum_{h \in L^{\prime} / L} \sum_{n>0} c_{f}^{-}(n, h) \int_{v=1}^{\infty} \sum_{X \in L_{-|\Delta| n, r h}} \chi_{\Delta}(X) \beta_{1 / 2}^{c}\left(4 \pi Q_{\Delta}(X) v\right) \exp \left(4 \pi Q_{\Delta}\left(X_{z^{\perp}}\right) v\right) v^{-s-1 / 2} d v,
$$

where the first two sums are finite. Hence, estimating

$$
\beta_{1 / 2}^{c}\left(4 \pi Q_{\Delta}(X) v\right) \leq 2 \exp \left(-4 \pi Q_{\Delta}(X) v\right)
$$

and using $Q_{\Delta}(X)=Q_{\Delta}\left(X_{z}\right)+Q_{\Delta}\left(X_{z^{\perp}}\right)$, it suffices to consider the integral

$$
\int_{v=1}^{\infty} \sum_{X \in L_{-|\Delta| n, r h}} \exp \left(-4 \pi Q_{\Delta}\left(X_{z}\right) v\right) v^{-\operatorname{Re}(s)-1 / 2} d v .
$$

For any $C \geq 0$ and any compact subset $K \subset \mathbb{H}$ the set

$$
\left\{X \in L_{-|\Delta| n, r h}: \exists z \in K \text { with }\left|Q_{\Delta}\left(X_{z}\right)\right| \leq C\right\}
$$

is finite, so if $z \in K \subset \mathbb{H} \backslash H_{\Delta, r}^{-}(f)$ then there is some $\varepsilon>0$ such that $Q_{\Delta}\left(X_{z}\right)>\varepsilon$ for all $X \in L_{-|\Delta| n, r h}$. We can now estimate

$$
\sum_{X \in L_{-|\Delta| n, r h}} \exp \left(-4 \pi Q_{\Delta}\left(X_{z}\right) v\right) \leq e^{-2 \pi \varepsilon v} e^{\pi n} \sum_{X \in L_{-|\Delta| n, r h}} \exp \left(-\pi\left(Q_{\Delta}\left(X_{z}\right)-Q_{\Delta}\left(X_{z^{\perp}}\right)\right)\right)
$$

for $v \geq 1$. The series on the right-hand side converges since $X \mapsto Q_{\Delta}\left(X_{z}\right)-Q_{\Delta}\left(X_{z^{\perp}}\right)$ is a positive definite quadratic form. In particular, the integral in (5) converges absolutely and locally uniformly for $s \in \mathbb{C}$ and $z \in \mathbb{H} \backslash H_{\Delta, r}^{-}(f)$. This shows that the regularized theta integral exists.

By similar arguments as above we see that all iterated partial derivatives of $\Phi_{\Delta, r}(f, z)$ converge absolutely and locally uniformly on $\mathbb{H} \backslash\left(H_{\Delta, r}^{+}(f) \cup H_{\Delta, r}^{-}(f)\right)$, so the Borcherds lift is a smooth function. The statement concerning the Laplacian can now be proven by interchanging $\Delta_{0}=\Delta_{0, z}$ with the integral, using the differential equation

$$
\Delta_{0, z} \Theta_{\Delta, r}(\tau, z)=4 v^{1 / 2} \overline{\Delta_{1 / 2, \tau} v^{-1 / 2} \overline{\Theta_{\Delta, r}(\tau, z)}},
$$

(which can be checked by a direct calculation) and then applying Stokes' theorem to move $\Delta_{1 / 2, \tau}$ from the theta function to $f(\tau) v^{-s}$ in the integral (compare [4], Lemma 4.3). It is easy to verify that the appearing boundary integrals vanish. By computing $\Delta_{1 / 2}\left(f(\tau) v^{-s}\right)$ explicitly and using that $f$ is harmonic, we obtain

$$
\Delta_{0} \Phi_{\Delta, r}(f, z)=-2 \operatorname{Res}_{s=0} \lim _{T \rightarrow \infty} \int_{\mathcal{F}_{T}}\left\langle f(\tau), \overline{\Theta_{\Delta, r}(\tau, z)}\right\rangle v^{-s} \frac{d u d v}{v^{2}} .
$$

We have seen above that the integral on the right-hand side is holomorphic at $s=0$ if $\Delta \neq 1$, and has a simple pole with residue $a_{f}^{+}(0,0)$ if $\Delta=1$, coming from the first integral in the first line of $\varphi(z, s)$. This shows the Laplace equation for $\Phi_{\Delta, r}(f, z)$, which also implies that the Borcherds lift is real analytic by a standard regularity result for elliptic differential equations.

The singularities of $\Phi_{\Delta, r}(f, z)$ can be determined using the following lemma with $n=$ $-Q_{\Delta}(X)$ and $t=-Q_{\Delta}\left(X_{z^{\perp}}\right)$.

Lemma 3.3. (1) The function

$$
I^{+}(t)=\int_{v=1}^{\infty} e^{-4 \pi t v} \frac{d v}{v}
$$

is real analytic for $t>0$ and has a singularity of type $-\log (t)$ at $t=0$. 
(2) For $n>0$ the function

$$
I_{n}^{-}(t)=\int_{v=1}^{\infty} \sqrt{v} \beta_{1 / 2}^{c}(-4 \pi n v) e^{-4 \pi t v} \frac{d v}{v}
$$

is real analytic for $t>n$ and has a singularity of type $n^{-1 / 2} \arcsin \left(\sqrt{\frac{n}{t}}\right)$ at $t=n$.

Proof. We follow the proof of [3], Lemma 6.1. Using partial integration and the fact that $\log (v)$ is integrable near $v=0$, we see that

$$
I^{+}(t) \approx 4 \pi t \int_{v=0}^{\infty} e^{-4 \pi t v} \log (v) d v=\int_{v=0}^{\infty} e^{-v} \log \left(\frac{v}{4 \pi t}\right) d v \approx-\log (t) .
$$

For $n>0$, we use that $\sqrt{v} \beta_{1 / 2}^{c}(-4 \pi n v)=O(\sqrt{v})$ as $v \rightarrow 0$ and compute

$$
\begin{aligned}
I_{n}^{-}(t) & \approx \int_{v=0}^{\infty}\left(2 \sqrt{v} \int_{w=0}^{1} e^{4 \pi n v w^{2}} d w\right) e^{-4 \pi t v} \frac{d v}{v} \\
& =\int_{w=0}^{1} \frac{1}{\sqrt{t-n w^{2}}} d w \\
& =n^{-1 / 2} \arcsin \left(\sqrt{\frac{n}{t}}\right) .
\end{aligned}
$$

This finishes the proof of the lemma and of Theorem 3.1.

\section{The Fourier expansion of the Borcherds Lift}

Next, we compute the Fourier expansion of the Borcherds lift. To this end, we first need to introduce a special function which captures the arcsin singularities of $\Phi_{\Delta, r}(f, z)$ along vertical geodesics.

For $a \geq 1$ and $\operatorname{Re}(s)>-1$ we define

$$
\arcsin _{s}\left(\frac{1}{\sqrt{a}}\right)=\int_{0}^{1} \frac{1}{\sqrt{a-t^{2}}}\left(\frac{1-t^{2}}{a-t^{2}}\right)^{s} d t
$$

The function $\arcsin _{s}$ is holomophic in $s$ and satisfies

$$
\arcsin _{0}(1 / \sqrt{a})=\arcsin (1 / \sqrt{a}) .
$$

The factor $\left(1-t^{2}\right)^{s}$ ensures that the integral converges at $a=1$ if $\operatorname{Re}(s) \geq 1 / 2$, and the factor $\left(a-t^{2}\right)^{s}$ in the denominator was added to make the estimate

$$
\left|\arcsin _{s}(1 / \sqrt{a})\right| \leq(a-1)^{-\operatorname{Re}(s)-1 / 2}
$$

for $a>1$ and $\operatorname{Re}(s)>0$ hold. Note that for $\operatorname{Re}(s)>-1$ we can write

$$
\arcsin _{s}\left(\frac{1}{\sqrt{a}}\right)=\frac{\sqrt{\pi} \Gamma(s+1)}{2 \Gamma(s+1 / 2)} B(1 / a ; s+1 / 2,1 / 2),
$$

where

$$
B(z ; \alpha, \beta)=\int_{0}^{z} u^{\alpha-1}(1-u)^{\beta-1} d u
$$

is the incomplete beta function. 
Lemma 4.1. For $z=x+i y \in \mathbb{H}$ and $\operatorname{Re}(s)>0$ we have the Fourier expansion

$$
\begin{aligned}
& \sum_{\ell \in \mathbb{Z}} \arcsin _{s}\left(\frac{y}{\sqrt{(x+\ell)^{2}+y^{2}}}\right)=y \frac{\sqrt{\pi} \Gamma(s)}{\Gamma(s+1 / 2)} \\
& \quad+2 y \frac{\sqrt{\pi}}{\Gamma(s+1 / 2)} \sum_{n \neq 0}(\pi|n| y)^{s}\left(\int_{0}^{1}\left(1-t^{2}\right)^{s / 2} K_{s}\left(2 \pi|n| y \sqrt{1-t^{2}}\right) d t\right) \cos (2 \pi n x),
\end{aligned}
$$

where $K_{s}$ denotes the $K$-Bessel function of order $s$. For $R e(s)>-1$ the series on the righthand side converges absolutely and locally uniformly in s. In particular, the left-hand side has a meromorphic continuation to $\operatorname{Re}(s)>-1$ with a simple pole at $s=0$.

Proof. The estimate (7) shows that the series on the left-hand side converges absolutely for $\operatorname{Re}(s)>0$. It is 1-periodic and even in $x$ and hence has a Fourier expansion of the form $\sum_{n \in \mathbb{Z}} a(n, y) \cos (2 \pi n x)$ with coefficients

$$
a(n, y)=\int_{-\infty}^{\infty} \arcsin _{s}\left(\frac{1}{\sqrt{(u / y)^{2}+1}}\right) \cos (2 \pi n u) d u
$$

We plug in the definition of $\arcsin _{s}$ and interchange the order of integration to find

$$
\begin{aligned}
a(n, y) & =\int_{0}^{1}\left(\int_{-\infty}^{\infty} \frac{\cos (2 \pi n u)}{\left((u / y)^{2}+1-t^{2}\right)^{s+1 / 2}} d u\right)\left(1-t^{2}\right)^{s} d t \\
& =y \int_{0}^{1}\left(\int_{-\infty}^{\infty} \frac{\cos \left(2 \pi n u y \sqrt{1-t^{2}}\right)}{\left(u^{2}+1\right)^{s+1 / 2}} d u\right) d t .
\end{aligned}
$$

For $n=0$ the inner integral can be evaluated as

$$
\int_{-\infty}^{\infty} \frac{1}{\left(u^{2}+1\right)^{s+1 / 2}} d u=\frac{\sqrt{\pi} \Gamma(s)}{\Gamma(s+1 / 2)}
$$

by a direct calculation using the definition of the Gamma function. For $n \neq 0$ we can replace $n$ by $|n|$, and then the inner integral can be computed using the representation

$$
K_{s}(x)=\frac{2^{s-1} \Gamma(s+1 / 2)}{\sqrt{\pi} x^{s}} \int_{-\infty}^{\infty} \frac{\cos (x u)}{\left(u^{2}+1\right)^{s+1 / 2}} d u
$$

which is valid for $\operatorname{Re}(s)>-1 / 2$ and $x>0$ (see [1, 9.6.25]). Note that $K_{s}=K_{-s}$.

The asymptotics $K_{0}(z) \sim-\log (z)$ and $K_{s}(z) \sim \frac{1}{2} \Gamma(s)\left(\frac{1}{2} z\right)^{-s}$ for $\operatorname{Re}(s)>0$ fixed as $z \rightarrow 0$ (see [1, 9.6.8, 9.6.9]) show that the integral in the series is holomorphic for $\operatorname{Re}(s)>-1$. This completes the proof. 


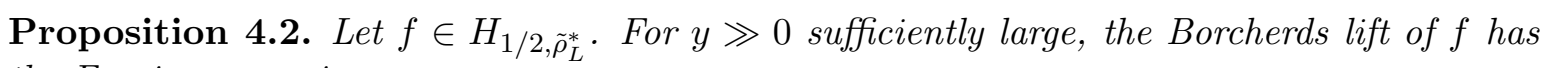
the Fourier expansion

$$
\begin{aligned}
\Phi_{\Delta, r}(f, z)= & -4 \sum_{m=1}^{\infty} c_{f}^{+}\left(|\Delta| m^{2} / 4 N, r m\right) \sum_{b(\Delta)}\left(\frac{\Delta}{b}\right) \log |1-e(m z+b / \Delta)| \\
& +2 \sum_{m=1}^{\infty} c_{f}^{-}\left(|\Delta| m^{2} / 4 N, r m\right)\left(\frac{|\Delta| m^{2}}{4 N}\right)^{-1 / 2} \sum_{b(\Delta)}\left(\frac{\Delta}{b}\right) \mathcal{F}(m z+b / \Delta) \\
& + \begin{cases}\sqrt{N} y\left(f, \theta_{1 / 2}\right)^{\mathrm{reg}}-c_{f}^{+}(0,0)\left(\log \left(4 \pi N y^{2}\right)+\Gamma^{\prime}(1)\right) & \text { if } \Delta=1, \\
-\sqrt{N} y c_{f}^{-}(0,0)\left(\log (4 \pi)-\log \left(N y^{2}\right)+\Gamma^{\prime}(1)\right) & \text { if } \Delta>1, \\
2 \sqrt{\Delta} L_{\Delta}(1)\left(c_{f}^{+}(0,0)+\sqrt{N} y c_{f}^{-}(0,0)\right) & \text { if } \Delta<0,\end{cases}
\end{aligned}
$$

where $\theta_{1 / 2}(\tau)=\sum_{h \in L^{\prime} / L} \sum_{n \in h+2 N \mathbb{Z}} e\left(n^{2} \tau / 4 N\right) \mathfrak{e}_{h}$ is the Jacobi theta function and $L_{\Delta}(s)=$ $\sum_{n \geq 1}\left(\frac{\Delta}{n}\right) n^{-s}$ for $\operatorname{Re}(s)>1$ is a Dirichlet L-function. Here the function $\mathcal{F}(z): \mathbb{H} \rightarrow \mathbb{R}$ is defined by

$$
\mathcal{F}(z)=\lim _{s \rightarrow 0}\left(\sum_{\ell \in \mathbb{Z}} \arcsin _{s}\left(\frac{y}{\sqrt{(x+\ell)^{2}+y^{2}}}\right)-y \frac{\sqrt{\pi} \Gamma(s)}{\Gamma(s+1 / 2)}\right),
$$

compare Lemma 4.1.

Remark 4.3. (1) The singularities of $\Phi_{\Delta, r}(f, z)$ at Heegner points and geodesics given by semi-circles centered at the real line are not reproduced in the Fourier expansion above, but the part involving the function $\mathcal{F}$ captures the singularities along vertical geodesics.

(2) By Dirichlet's class number formula we have

$$
L_{\Delta}(1)=\frac{1}{\sqrt{\Delta}} h(\Delta) \log \left(\epsilon_{\Delta}\right)=\frac{1}{2} \operatorname{tr}_{1}(\Delta)
$$

for $\Delta>1$, where $h(\Delta)$ is the narrow class number of $\mathbb{Q}(\sqrt{\Delta}), \epsilon_{\Delta}$ is the smallest unit $>1$ of norm 1 , and $\operatorname{tr}_{1}(\Delta)$ is the $\Delta$-th trace of the constant 1 function as defined in the introduction.

Proof of Proposition 4.2. The proof follows the arguments of [8], Theorem 5.3. First, by [8], Theorem 4.8, we can write

$$
\begin{aligned}
v^{-1 / 2} \overline{\Theta_{\Delta, r}(\tau, z)}= & \delta_{\Delta=1} \frac{\sqrt{N} y}{\sqrt{|\Delta|}} \theta_{1 / 2}(\tau) \\
& +\left.\frac{\sqrt{N} y}{\sqrt{|\Delta|}} \sum_{n \geq 1} \sum_{M \in \tilde{\Gamma}_{\infty} \backslash \tilde{\Gamma}}\left[\exp \left(-\frac{\pi n^{2} N y^{2}}{|\Delta| v}\right) \Xi(\tau, \mu, n, 0)\right]\right|_{1 / 2, \tilde{\rho}_{L}^{*}} M,
\end{aligned}
$$

where $\mu=\left(\begin{array}{cc}x & -x^{2} \\ -1 & -x\end{array}\right)$ and

$$
\Xi(\tau, \mu, n, 0)=\left(\frac{\Delta}{n}\right) \bar{\varepsilon} \sqrt{|\Delta|} \sum_{h \in K^{\prime} / K} \sum_{\substack{X \in K+r h \\ Q(X) \equiv \Delta Q(h)(\Delta)}} e\left(-Q_{\Delta}(X) \tau+n(X, \mu)_{\Delta}\right) \mathfrak{e}_{h},
$$


with $\varepsilon=1$ if $\Delta>0$ and $\varepsilon=i$ if $\Delta<0$. Further, $K$ denotes the one-dimensional negative definite sublattice

of $L$. Its dual lattice is given by

$$
K=\left\{\left(\begin{array}{cc}
b & 0 \\
0 & -b
\end{array}\right): b \in \mathbb{Z}\right\}
$$

$$
K^{\prime}=\left\{\left(\begin{array}{cc}
b / 2 N & 0 \\
0 & -b / 2 N
\end{array}\right): b \in \mathbb{Z}\right\} .
$$

Inserting this into the definition of the theta lift, the unfolding argument yields

$$
\Phi_{\Delta, r}(f, z)=\delta_{\Delta=1} \frac{\sqrt{N} y}{\sqrt{|\Delta|}}\left(f, \theta_{1 / 2}\right)^{\mathrm{reg}}+\mathrm{CT}_{s=0} \Phi_{\Delta, r}^{0}(f, z, s),
$$

where

$$
\Phi_{\Delta, r}^{0}(f, z, s)=\frac{2 \sqrt{N} y}{\sqrt{|\Delta|}} \sum_{n \geq 1} \int_{v=0}^{\infty} \int_{u=0}^{1} \exp \left(-\frac{\pi n^{2} N y^{2}}{|\Delta| v}\right)\langle f, \Xi(\tau, \mu, n, 0)\rangle d u \frac{d v}{v^{s+3 / 2}} .
$$

The unfolding is justified for $y \gg 0$ by the same arguments as in [3], Theorem 7.1. Let us write

$$
f(\tau)=\sum_{h \in L^{\prime} / L} \sum_{n \in \mathbb{Q}} c_{f}(n, h, v) e(n \tau) \mathfrak{e}_{h}
$$

for the Fourier expansion of $f$ for the moment. Since $\Delta$ is fundamental, the conditions $X \in K+r h$ and $Q(X) \equiv \Delta Q(h) \bmod \Delta$ are equivalent to $X=\Delta X^{\prime}$ and $r X^{\prime} \in K+h$ for some $X^{\prime} \in K^{\prime}$. Plugging in the definition of $\Xi(\tau, n, \mu, 0)$, and evaluating the integral over $u$, we obtain

$$
\begin{aligned}
\Phi_{\Delta, r}^{0}(f, z, s)= & 2 \sqrt{N} y \varepsilon \sum_{X \in K^{\prime}} \sum_{n \geq 1}\left(\frac{\Delta}{n}\right) e(-\operatorname{sgn}(\Delta) n(X, \mu)) \\
& \times \int_{v=0}^{\infty} c_{f}(-|\Delta| Q(X), r X, v) \exp \left(-\frac{\pi n^{2} N y^{2}}{|\Delta| v}+4 \pi|\Delta| Q(X) v\right) \frac{d v}{v^{s+3 / 2}} .
\end{aligned}
$$

Now we use the explicit form of the Fourier coefficients of $f$. The summand for $X=0$ in $\Phi_{\Delta, r}^{0}(f, z, s)$ is given by

$$
\begin{gathered}
2 \sqrt{N} y \varepsilon \sum_{n \geq 1}\left(\frac{\Delta}{n}\right) \int_{v=0}^{\infty}\left(c_{f}^{+}(0,0)+c_{f}^{-}(0,0) v^{1 / 2}\right) \exp \left(-\frac{\pi n^{2} N y^{2}}{|\Delta| v}\right) \frac{d v}{v^{s+3 / 2}} \\
=2 \varepsilon\left(N y^{2}\right)^{-s}\left(c_{f}^{+}(0,0)\left(\frac{\pi}{|\Delta|}\right)^{-s-1 / 2} \Gamma(s+1 / 2) L_{\Delta}(2 s+1)\right. \\
\left.+\sqrt{N} y c_{f}^{-}(0,0)\left(\frac{\pi}{|\Delta|}\right)^{-s} \Gamma(s) L_{\Delta}(2 s)\right) .
\end{gathered}
$$

For $\Delta<0$ the harmonic Maass form $f$ transforms with $\rho_{L}$, which implies that its zero component vanishes, so $c_{f}^{ \pm}(0,0)=0$. For $\Delta>0$ the completed Dirichlet $L$-function

$$
\Lambda_{\Delta}(s)=(\pi / \Delta)^{-s / 2} \Gamma(s / 2) L_{\Delta}(s)
$$

satisfies the functional equation $\Lambda_{\Delta}(1-s)=\Lambda_{\Delta}(s)$. It is holomorphic at $s=1$ if $\Delta>1$. Taking the constant term at $s=0$, we get the contribution in the large bracket in the proposition. 
For $X \in K^{\prime}$ with $X \neq 0$ we have $-|\Delta| Q(X)>0$. We can write

$$
c_{f}(n, h, v)=c_{f}^{+}(n, h)+c_{f}^{-}(n, h) \sqrt{v} \beta_{1 / 2}^{c}(-4 \pi n v)
$$

for $n>0$. The contribution coming from the coefficients $c_{f}^{+}(n, h)$ can be computed as in [8], Theorem 5.2, and yields the first line of the Fourier expansion. Plugging in the definition of $\beta_{1 / 2}^{c}(s)$, it remains to compute

$$
\begin{aligned}
& 4 \sqrt{N} y \varepsilon \sum_{\substack{X \in K^{\prime} \\
X \neq 0}} c_{f}^{-}(-|\Delta| Q(X), r X) \sum_{n \geq 1}\left(\frac{\Delta}{n}\right) e(-\operatorname{sgn}(\Delta) n(X, \mu)) \\
& \quad \times \int_{v=0}^{\infty}\left(\int_{w=0}^{1} \exp \left(-4 \pi|\Delta| Q(X) w^{2} v\right) d w\right) \exp \left(-\frac{\pi n^{2} N y^{2}}{|\Delta| v}+4 \pi|\Delta| Q(X) v\right) \frac{d v}{v} .
\end{aligned}
$$

If we change the order of integration, the inner integral can be computed in terms of the $K$-Bessel function by [13, (3.471.9)], giving

$$
\int_{v=0}^{\infty} \exp \left(4 \pi|\Delta| Q(X)\left(1-w^{2}\right) v-\frac{\pi n^{2} N y^{2}}{|\Delta| v}\right) \frac{d v}{v}=2 K_{0}\left(2 \pi y|n| \sqrt{-4 N Q(X)\left(1-w^{2}\right)}\right) .
$$

Write $X=\left(\begin{array}{cc}m / 2 N & 0 \\ 0 & -m / 2 N\end{array}\right) \in K^{\prime} \backslash\{0\}$ with $m \in \mathbb{Z}, m \neq 0$. Then $-Q(X)=m^{2} / 4 N$ and $-(X, \mu)=m x$. We use the evaluation of the Gauss sum

$$
\sum_{b(\Delta)}\left(\frac{\Delta}{b}\right) e(b n /|\Delta|)=\varepsilon\left(\frac{\Delta}{n}\right) \sqrt{|\Delta|} .
$$

Then the expression in (9) becomes

$$
\begin{aligned}
& 2 \sum_{m=1}^{\infty} a_{f}\left(|\Delta| m^{2} / 4 N, r m\right)\left(\frac{|\Delta| m^{2}}{4 N}\right)^{-1 / 2} \sum_{b(\Delta)}\left(\frac{\Delta}{b}\right) \\
& \quad \times 2 m y \sum_{n \neq 0} e(n(m x+b / \Delta)) \int_{0}^{1} K_{0}\left(2 \pi m y|n| \sqrt{1-w^{2}}\right) d w .
\end{aligned}
$$

By Lemma 4.1 the second line agrees with $\mathcal{F}(m z+b / \Delta)$, which finishes the proof.

If the coefficients $c_{f}^{+}(n, h)$ vanish for $n<0$, then $\Phi_{\Delta, r}(f, z)$ does not have singularities at Heegner points, and extends to a continuous function on $\mathbb{H}$ which is not differentiable along the geodesics in $H_{\Delta, r}^{-}(f)$. In this case, we can derive the Fourier expansion of $\Phi_{\Delta, r}(f, z)$ on $\mathbb{H}$, without assuming $y \gg 0$ to be large enough.

Corollary 4.4. Let $f \in H_{1 / 2, \tilde{\rho}_{L}^{*}}$, and suppose that $c_{f}^{+}(n, h)=0$ for all $n<0$ and $h \in L^{\prime} / L$. Then the Fourier expansion of the Borcherds lift $\Phi_{\Delta, r}(f, z)$ on $\mathbb{H}$ is given by the formula from Proposition 4.2 plus the expression

$$
\text { (11) }-2 \sum_{h \in L^{\prime} / L} \sum_{n>0} c_{f}^{-}(n, h) n^{-1 / 2} \sum_{\substack{X \in L_{-|\Delta| n, r h} \\ a \neq 0}} \chi_{\Delta}(X) \mathbf{1}_{X}(z)\left(\arctan \left(\frac{\sqrt{4|\Delta| n}}{-\operatorname{sgn}(a) p_{X}(z)}\right)+\frac{\pi}{2}\right),
$$

where $\mathbf{1}_{X}(z)$ denotes the characteristic function of the bounded component of $\mathbb{H} \backslash c_{X}$. 
Remark 4.5. (1) Recall that for $X=\left(\begin{array}{cc}-b / 2 N & -c / N \\ a & b / 2 N\end{array}\right) \in L^{\prime}$ we defined

$$
p_{X}(z)=-\frac{a N|z|^{2}+b x+c}{y \sqrt{N}}
$$

which vanishes exactly along the geodesic $c_{X}$. Further, if $a \neq 0$ then a point $z$ lies inside the bounded component of $\mathbb{H} \backslash c_{X}$ if and only if $\operatorname{sgn}(a) p_{X}(z)>0$. In particular, we see that if $z \in \mathbb{H} \backslash c_{X}$ approaches $c_{X}$, then the expression

$$
\mathbf{1}_{X}(z)\left(\arctan \left(\frac{\sqrt{4|\Delta| n}}{-\operatorname{sgn}(a) p_{X}(z)}\right)+\frac{\pi}{2}\right)
$$

goes to 0 . In this sense, the above Fourier expansion is defined on all of $\mathbb{H}$.

(2) The sum in (11) is locally finite since for fixed $n$ each point $z$ lies in the bounded component of $\mathbb{H} \backslash c_{X}$ for only finitely many $X \in L_{-|\Delta| n, r h}$ with $a \neq 0$.

Proof. Let $\tilde{\Phi}_{\Delta, r}(f, z)$ denote $\Phi_{\Delta, r}(f, z)$ minus the expression in (11). Then we have $\tilde{\Phi}_{\Delta, r}(f, z)=$ $\Phi_{\Delta, r}(f, z)$ for $y \gg 0$ large enough since the imaginary parts of points lying on geodesics $c_{X}$ for $X \in L_{-|\Delta| n, r h}$ with $a \neq 0$ are bounded by a constant depending on $n$, and the sum over $n$ is finite.

Further, for $a \neq 0$ and $z \notin c_{X}$ we can write

$$
\begin{aligned}
& -2 \cdot \mathbf{1}_{X}(z)\left(\arctan \left(\frac{\sqrt{4|\Delta| n}}{-\operatorname{sgn}(a) p_{X}(z)}\right)+\frac{\pi}{2}\right) \\
& =\arctan \left(\frac{\sqrt{4|\Delta| n}}{\left|p_{X}(z)\right|}\right)-\left(\arctan \left(\frac{\sqrt{4|\Delta| n}}{-\operatorname{sgn}(a) p_{X}(z)}\right)+\mathbf{1}_{X}(z) \pi\right) \\
& =\arctan \left(\sqrt{\frac{Q_{\Delta}(X)}{-Q_{\Delta}\left(X_{z}\right)}}\right)-\operatorname{arccot}\left(\frac{-\operatorname{sgn}(a) p_{X}(z)}{\sqrt{4|\Delta| n}}\right) .
\end{aligned}
$$

Using that the function arccot is real analytic at the origin, and the shape of the singularities of $\Phi_{\Delta, r}(f, z)$ determined in Theorem 3.1, we see that $\tilde{\Phi}_{\Delta, r}(f, z)$ extends to a real analytic on all of $\mathbb{H}$. In particular, the Fourier expansion of $\Phi_{\Delta, r}(f, z)$ given in Proposition 4.2, which a priori only converges for $y \gg 0$ sufficiently large, is also the Fourier expansion of the real analytic function $\tilde{\Phi}_{\Delta, r}(f, z)$ on all of $\mathbb{H}$, and hence converges on all of $\mathbb{H}$. We obtain the stated Fourier expansion.

\section{The Derivative of THE Borcherds LifT}

We consider the derivative

$$
\Phi_{\Delta, r}^{\prime}(f, z)=\frac{\partial}{\partial z} \Phi_{\Delta, r}(f, z)
$$

of the Borcherds lift.

Theorem 5.1. Let $f \in H_{1 / 2, \tilde{\rho}_{L}^{*}}$. The derivative $\Phi_{\Delta, r}^{\prime}(f, z)$ of the Borcherds lift is harmonic on $\mathbb{H} \backslash\left(H_{\Delta, r}^{+}(f) \cup H_{\Delta, r}^{-}(f)\right)$ and transforms like a modular form of weight 2 under $\Gamma_{0}(N)$. If $\Delta \neq 1$ or if $c_{f}^{+}(0,0)=0$, then $\Phi_{\Delta, r}^{\prime}(f, z)$ is holomorphic on its domain. 
At a point $z_{0} \in H_{\Delta, r}^{+}(f) \cup H_{\Delta, r}^{-}(f)$ it has a singularity of type

$$
\begin{aligned}
& i \sqrt{N} \sum_{h \in L^{\prime} / L} \sum_{n<0} c_{f}^{+}(n, h) \sum_{\substack{X \in L_{-}|\Delta| n, r h \\
z_{0}=z_{X}}} \chi_{\Delta}(X) \frac{p_{X}(z)}{Q_{X}(z)} \\
& +i \sqrt{N|\Delta|} \sum_{h \in L^{\prime} / L} \sum_{n>0} c_{f}^{-}(n, h) \sum_{\substack{X \in L_{-}|\Delta| n, r h \\
z_{0} \in c_{X}}} \chi_{\Delta}(X) \frac{\operatorname{sgn}\left(p_{X}(z)\right)}{Q_{X}(z)} .
\end{aligned}
$$

Proof. The analytic properties of $\Phi_{\Delta, r}^{\prime}(f, z)$ follow from the Laplace equation in Theorem 3.1 and the formula $\Delta_{0}=-4 y^{2} \frac{\partial}{\partial \bar{z}} \frac{\partial}{\partial z}$. The types of singularities of $\Phi_{\Delta, r}^{\prime}(f, z)$ are obtained as the derivatives of the types of singularities of $\Phi_{\Delta, r}(f, z)$.

Remark 5.2. Let $X=\left(\begin{array}{cc}-b / 2 N & -c / N \\ a & b / 2 N\end{array}\right) \in L_{-|\Delta| n, r h}$. For $n<0$ we have

$$
Q_{X}(z)=a N z^{2}+b z+c=0
$$

exactly for the Heegner point $z=z_{X}$. Hence $\Phi_{\Delta, r}^{\prime}(f, z)$ has simple poles at the Heegner points in $H_{\Delta, r}^{+}(f)$. For $n>0$ the sign of

$$
p_{X}(z)=-\frac{a N|z|^{2}+b x+c}{y \sqrt{N}}
$$

changes if $z$ crosses the geodesic $c_{X}$. This means that $\Phi_{\Delta, r}^{\prime}(f, z)$ has jump singularities along the geodesics in $H_{\Delta, r}^{-}(f)$.

Proposition 5.3. Let $f \in H_{1 / 2, \tilde{\rho}_{L}^{*}}$. For $y \gg 0$ sufficiently large we have the Fourier expansion

$$
\begin{aligned}
\Phi_{\Delta, r}^{\prime}(f, z)= & 4 \pi i \sqrt{|\Delta|} \bar{\varepsilon} \sum_{n=1}^{\infty}\left(\sum_{d \mid n}\left(\frac{\Delta}{n / d}\right) d c_{f}^{+}\left(|\Delta| d^{2} / 4 N, r d\right)\right) e(n z) \\
& +2 \sum_{m=1}^{\infty} c_{f}^{-}\left(|\Delta| m^{2} / 4 N, r m\right)\left(\frac{|\Delta|}{4 N}\right)^{-1 / 2} \sum_{b(\Delta)}\left(\frac{\Delta}{b}\right) \mathcal{F}^{\prime}(m z+b / \Delta) \\
& + \begin{cases}-\frac{i \sqrt{N}}{2}\left(f, \theta_{1 / 2}\right)^{\mathrm{reg}}+\frac{i}{y} c_{f}^{+}(0,0) & \text { if } \Delta=1, \\
+\frac{i}{2} \sqrt{N} c_{f}^{-}(0,0)\left(\log (4 \pi)-\log \left(N y^{2}\right)-2+\Gamma^{\prime}(1)\right) & \text { if } \Delta>1, \\
-i \sqrt{N \Delta} L_{\Delta}(1) c_{f}^{-}(0,0) & \text { if } \Delta<0 . \\
0 & \end{cases}
\end{aligned}
$$

where $\varepsilon=1$ if $\Delta>0$ if $\varepsilon=i$ for $\Delta<0$, and

$$
\mathcal{F}^{\prime}(z)=-\frac{i}{2} \lim _{s \rightarrow 0}\left(y^{2 s} \Gamma(s+1) \sum_{\ell \in \mathbb{Z}} \frac{\operatorname{sgn}(x+\ell)(\bar{z}+\ell)}{|z+\ell|^{2 s+2}}-\Gamma(s)\right) .
$$

Proof. The derivative of $\mathcal{F}(z)$ can be computed most easily using the representation (8) of $\arcsin _{s}$ as an incomplete beta function. Using the formula (10) for the Gauss sum, the calculation of the remaining derivatives is straightforward. 
Again, we consider the special case that $c_{f}^{+}(n, h)=0$ for all $n<0$.

Corollary 5.4. Let $f \in H_{1 / 2, \tilde{\rho}_{L}^{*}}$, and suppose that $c_{f}^{+}(n, h)=0$ for all $n<0$ and $h \in L^{\prime} / L$. Then the Fourier expansion of the derivative $\Phi_{\Delta, r}^{\prime}(f, z)$ of the Borcherds lift on $\mathbb{H} \backslash H_{\Delta, r}^{-}(f)$ is given by the formula from Proposition 5.3 plus the expression

$$
-2 i \sqrt{|\Delta| N} \sum_{h \in L^{\prime} / L} \sum_{n>0} c_{f}^{-}(n, h) \sum_{\substack{X \in L_{-|\Delta| n, r h} \\ a \neq 0}} \chi_{\Delta}(X) \frac{\mathbf{1}_{X}(z) \operatorname{sgn}(a)}{Q_{X}(z)},
$$

where $\mathbf{1}_{X}(z)$ denotes the characteristic function of the bounded component of $\mathbb{H} \backslash c_{X}$.

Proof. This can either be proved by similar arguments as in the proof of Corollary 4.4, or by computing the derivative of the expression (11).

\section{Applications: Modular integrals With Rational Period functions and BORCHERDS PRODUCTS OF HARMONIC MAASS FORMS}

For simplicity, we assume in this section that $N$ is square free. Then the cusps of $\Gamma_{0}(N)$ can be represented by the fractions $1 / c$ with $c \mid N$. Note that $\infty$ corresponds to $1 / N$. The width of $1 / c$ is given by $\alpha_{1 / c}=N / c$. We choose the matrix $\sigma_{1 / c} \in \mathrm{SL}_{2}(\mathbb{Z})$ sending $\infty$ to $1 / c$ in the form

$$
\sigma_{1 / c}=\left(\begin{array}{cc}
1 & \beta \\
c & N \gamma / c
\end{array}\right)
$$

where $\beta, \gamma \in \mathbb{Z}$ are such that $N \gamma / c-c \beta=1$. Then we can take the Atkin-Lehner involution corresponding to $N / c$ as

$$
W_{N / c}=\sigma_{1 / c}\left(\begin{array}{cc}
N / c & 0 \\
0 & 1
\end{array}\right) \text {. }
$$

We see that $W_{N / c} \infty=1 / c$, so the Atkin-Lehner involutions act transitively on the cusps. Further, the expansion at the cusp $1 / c$ of a function $F$, which is modular of weight $k \in \mathbb{Z}$, is given by

$$
\left(\left.F\right|_{k} \sigma_{1 / c}\right)(z)=(c / N)^{k / 2} \cdot\left(\left.F\right|_{k} W_{N / c}\right)(c z / N)
$$

Since

and consequently

$$
\left.\Phi_{\Delta, r}(f, z)\right|_{0} W_{N / c}=\Phi_{\Delta, r}\left(f^{w_{N / c}}, z\right)
$$

$$
\left.\Phi_{\Delta, r}^{\prime}(f, z)\right|_{2} W_{N / c}=\Phi_{\Delta, r}^{\prime}\left(f^{w_{N / c}}, z\right),
$$

the expansion of $\Phi_{\Delta, r}^{\prime}(f, z)$ at the cusp $1 / c$ is essentially given by $\Phi_{\Delta, r}^{\prime}\left(f^{w_{N / c}}, z\right)$.

6.1. Modular integrals with rational period functions. As an application of our extension of the Borcherds lift, we construct modular integrals of weight 2 for $\Gamma_{0}(N)$ with rational period functions from harmonic Maass forms of weight 1/2. Following Knopp [14], we call a holomorphic function $F: \mathbb{H} \rightarrow \mathbb{C}$ a modular integral of weight $k \in \mathbb{Z}$ for $\Gamma_{0}(N)$ with rational period functions if

$$
q_{M}(z)=F(z)-\left(\left.F\right|_{k} M\right)(z)
$$

is a rational function of $z$ for each $M \in \Gamma_{0}(N)$, and if $F$ is holomorphic at the cusps of $\Gamma_{0}(N)$, in the sense that $\lim _{y \rightarrow \infty}\left(\left.F\right|_{k} M\right)(z)$ exists for every $M \in \mathrm{SL}_{2}(\mathbb{Z})$. Then the map 
$M \mapsto q_{M}$ defines a weight $k$ cocycle for $\Gamma_{0}(N)$ with values in the rational functions which are holomorphic on $\mathbb{H}$, i.e., it satisfies

$$
q_{M M^{\prime}}=\left.q_{M}\right|_{k} M^{\prime}+q_{M^{\prime}}
$$

for all $M, M^{\prime} \in \Gamma_{0}(N)$. Conversely, it follows from a more general result of Knopp [14 that every such cocycle admits a holomorphic modular integral. Knopp's modular integrals are Poincaré series built from the cocycles. It was shown in 9] and 10 that certain generating series of (traces of) cycle integrals of weakly holomorphic modular functions for $\mathrm{SL}_{2}(\mathbb{Z})$ are modular integrals of weight 2 with rational period functions. Using the Borcherds lift we generalize their construction to higher level.

Proposition 6.1. Let $\Delta \neq 1$ be a fundamental discriminant. Let $f \in H_{1 / 2, \tilde{\rho}_{L}^{*}}$ with $c_{f}^{+}(n, h)=$ 0 for all $n<0$ and $h \in L^{\prime} / L$. Further, assume that $c_{f}^{-}\left(|\Delta| m^{2} / 4 N, r m\right)=0$ for all $m \in \mathbb{Z}, m>$ 0 . Then the function

$$
F_{\Delta, r}(f, z)=-\frac{1}{4 \pi} L_{\Delta}(1) c_{f}^{-}(0,0)+\frac{\bar{\varepsilon}}{\sqrt{N}} \sum_{n=1}^{\infty}\left(\sum_{d \mid n}\left(\frac{\Delta}{n / d}\right) d c_{f}^{+}\left(|\Delta| d^{2} / 4 N, r d\right)\right) e(n z)
$$

is holomorphic on $\mathbb{H}$ and at the cusps of $\Gamma_{0}(N)$, and satisfies the transformation rule

$$
\left.F_{\Delta, r}(f, z)\right|_{2} M-F_{\Delta, r}(f, z)=-\frac{1}{\pi} \sum_{h \in L^{\prime} / L} \sum_{n>0} c_{f}^{-}(n, h) \sum_{\substack{X \in L_{-}|\Delta| n, r h \\ a_{M X}<0<a_{X}}} \frac{\chi_{\Delta}(X)}{Q_{X}(z)}
$$

for all $M \in \Gamma_{0}(N)$, where $a_{X}$ denotes the a entry of $X$. In particular, $F_{\Delta, r}(f, z)$ is a modular integral of weight 2 for $\Gamma_{0}(N)$.

Remark 6.2. (1) The requirement $c_{f}^{-}\left(|\Delta| m^{2} / 4 N, r m\right)=0$ for all $m \in \mathbb{Z}, m>0$, ensures that $\Phi_{\Delta, r}^{\prime}(f, z)$ does not have singularities along vertical geodesics, and implies that the second line of the Fourier expansion in Proposition 5.3 vanishes.

(2) The proof of the transformation behaviour works for arbitrary positive integers $N$, but the assumption that $N$ is square free is used to obtain the Fourier expansions of $\Phi_{\Delta, r}^{\prime}(f, z)$ at different cusps via Atkin-Lehner operators. One could compute the expansion at a cusp $\ell$ by choosing an appropriate sublattice $K_{\ell}$ instead of $K$ in Proposition 4.2 and modify the computation of the expansion at $\infty$ correspondingly. However, the above result is certainly true without the assumption that $N$ is square free, but the computations become much more technical.

Proof of Proposition 6.1. Let $z \in \mathbb{H} \backslash H_{\Delta, r}^{-}(h)$, and let

$$
F_{\Delta, r}^{*}(f, z)=-\frac{1}{2 \pi} \sum_{h \in L^{\prime} / L} \sum_{n>0} c_{f}^{-}(n, h) \sum_{\substack{X \in L_{-|\Delta| n, r h} \\ a \neq 0}} \chi_{\Delta}(X) \frac{\mathbf{1}_{X}(z) \operatorname{sgn}(a)}{Q_{X}(z)} .
$$

By Corollary 5.4 we have

$$
\Phi_{\Delta, r}^{\prime}(f, z)=4 \pi i \sqrt{N|\Delta|}\left(F_{\Delta, r}(f, z)+F_{\Delta, r}^{*}(f, z)\right) .
$$

Since $\Phi_{\Delta, r}^{\prime}(f, z)$ transforms like a modular form of weight 2 for $\Gamma_{0}(N)$, we obtain

$$
\left.F_{\Delta, r}(f, z)\right|_{2} M-F_{\Delta, r}(f, z)\left|=-F_{\Delta, r}^{*}(f, z)\right|_{2} M+F_{\Delta, r}^{*}(f, z) .
$$


Using $\left.Q_{X}(z)\right|_{-2} M=Q_{M^{-1} X}(z)$, we obtain that the right-hand side of the last formula equals

$$
-\frac{1}{2 \pi} \sum_{h \in L^{\prime} / L} \sum_{n>0} c_{f}^{-}(n, h) \sum_{\substack{X \in L_{-|| \mid n, r h} \\ a \neq 0}} \chi_{\Delta}(X) \frac{\mathbf{1}_{X}(z) \operatorname{sgn}\left(a_{X}\right)-\mathbf{1}_{M X}(M z) \operatorname{sgn}\left(a_{M X}\right)}{Q_{X}(z)} .
$$

The characteristic functions $\mathbf{1}_{X}$ and $\mathbf{1}_{M X}$ are related by

$$
\mathbf{1}_{M X}(M z)= \begin{cases}\mathbf{1}_{X}(z), & \text { if } a_{X} \cdot a_{M X}>0 \\ 1-\mathbf{1}_{X}(z), & \text { if } a_{X} \cdot a_{M X}<0\end{cases}
$$

In particular, all summands with $a_{X} \cdot a_{M X}>0$ cancel out. In the remaining sum over $X$ with $a_{X} \cdot a_{M X}<0$, we replace $X$ with $-X$ if $a_{X}<0$, giving a factor 2 . This proves the transformation behaviour of $F_{\Delta, r}(f, z)$ for $z \in \mathbb{H} \backslash H_{\Delta, r}^{-}(f)$. Since all the functions appearing in the transformation formula are holomorphic on $\mathbb{H}$, we obtain the transformation law by analytic continuation.

Using $\left.\Phi_{\Delta, r}^{\prime}(f, z)\right|_{2} W_{d}=\Phi_{\Delta, r}^{\prime}\left(f^{w_{d}}, z\right)$ we obtain

$$
\left.F_{\Delta, r}(f, z)\right|_{2} W_{d}=F_{\Delta, r}\left(f^{w_{d}}, z\right)+F_{\Delta, r}^{*}\left(f^{w_{d}}, z\right)+F_{\Delta, r}^{*}(f, z) \mid W_{d} .
$$

Since $F_{\Delta, r}\left(f^{w_{d}}, z\right)$ is holomorphic at $\infty$, and $F_{\Delta, r}^{*}\left(f^{w_{d}}, z\right)$ and $\left.F_{\Delta, r}^{*}(f, z)\right|_{2} W_{d}$ vanish as $y \rightarrow \infty$, we see that $F_{\Delta, r}(f, z)$ is holomorphic at the cusps.

Example 6.3. Let $\Delta>1$. We apply Proposition 6.1 to a harmonic Maass form $f \in H_{1 / 2, \rho_{L}^{*}}$ arising as the image of the regularized theta lift studied by Bruinier, Funke and Imamoglu in [7] of a harmonic Maass form $F \in H_{0}^{+}\left(\Gamma_{0}(N)\right)$. We assume that the constant coefficients $a_{\ell}^{+}(0)$ of $F$ vanish at all cusps. By Theorem 4.1 in [7] the Fourier expansion of the $h$-th component of $f$ is given by

$$
\begin{aligned}
f_{h}(\tau)= & -2 \operatorname{tr}_{F}(0, h) \sqrt{v} \\
& +\sum_{n<0} \operatorname{tr}_{F}(-n, h) \sqrt{v} \beta_{1 / 2}(4 \pi|n| v) e(n \tau) \\
& +\sum_{n>0} \frac{\sqrt{N}}{\pi} \operatorname{tr}_{F}(-n, h) e(n \tau) \\
& +\sum_{n>0} \operatorname{tr}_{F}^{c}\left(-n^{2} / 4 N, h\right) \sqrt{v} \beta_{1 / 2}^{c}\left(-4 \pi n^{2} v / 4 N\right) e\left(n^{2} \tau / 4 N\right),
\end{aligned}
$$

with the traces

$$
\operatorname{tr}_{F}(-n, h)=\left\{\begin{array}{cl}
\sum_{X \in \Gamma_{0}(N) \backslash L_{-n, h}} \frac{1}{\left|\bar{\Gamma}_{X}\right|} F\left(z_{X}\right), & n<0, \\
-\delta_{0, h} \frac{1}{2 \pi} \int_{\Gamma_{0}(N) \backslash \mathbb{H}}^{\mathrm{reg}} F(z) \frac{d x d y}{y^{2}}, & n=0, \\
\sum_{X \in \Gamma_{0}(N) \backslash L_{-n, h}} \int_{\Gamma_{X} \backslash c_{X}}^{\mathrm{reg}} F(z) \frac{d z}{Q(z, 1)}, & n>0,
\end{array}\right.
$$

and the so-called complementary trace $\operatorname{tr}_{F}^{c}\left(-n^{2} / 4 N, h\right)$, which is defined in [7, Section 3 . Our definition of the traces of cycle integrals equals $\pi / \sqrt{N}$ times the traces of cycle integrals defined in [7, and the traces for $|n| / N$ being a square need to be regularized as explained in [7, Section 3. Note that the trace of index 0 and the complementary trace can be evaluated 
explicitly in terms of the principal parts of $F$ at the cusps of $\Gamma_{0}(N)$, see [6], Remark 4.9, and that the complementary trace is nonzero only for finitely many $n$, see [6], Proposition 4.7. Observe that $c_{f}^{+}(n, h)=0$ for $n<0$ and $c_{f}^{-}\left(\Delta m^{2} / 4 N, r m\right)=0$ for $m \in \mathbb{Z}, m>0$, if $\Delta>1$. The Duke-Imamoglu-Tóth harmonic Maass form $h(\tau)=h_{1}(\tau)$ from the introduction can be constructed as the Bruinier-Funke-Imamoglu lift of $\frac{1}{2} J$.

By Proposition 6.1, for $\Delta>1$ a fundamental discriminant the function

$$
F_{\Delta, r}(f, z)=\frac{1}{2 \pi} L_{\Delta}(1) \operatorname{tr}_{F}(0,0)+\frac{1}{\pi} \sum_{n=1}^{\infty}\left(\sum_{d \mid n}\left(\frac{\Delta}{n / d}\right) d \operatorname{tr}_{F}\left(-\Delta d^{2} / 4 N, r d\right)\right) e(n z)
$$

is a holomorphic function on $\mathbb{H}$, which transforms under the weight 2 slash operation of $M \in \Gamma_{0}(N)$ by

$$
\left.F_{\Delta, r}(f, z)\right|_{2} M-F_{\Delta, r}(f, z)=-\frac{1}{\pi} \sum_{h \in L^{\prime} / L} \sum_{n>0} \operatorname{tr}_{F}^{c}\left(-n^{2} / 4 N, h\right) \sum_{\substack{X \in L_{-\Delta n^{2} / 4 N, r h} \\ a_{M X}<0<a_{X}}} \frac{1}{Q_{X}(z)} .
$$

Since $\chi_{\Delta}(X)=1$ for $X \in L_{-\Delta n^{2} / 4 N, r h}$ we dropped it from the notation.

In the special case $N=1$ and $F=\frac{1}{2} J$ (with $\operatorname{tr}_{J}(0,0)=4$ and $\operatorname{tr}_{J}^{c}(-1 / 4,1)=2$ ) we recover the transformation behaviour of the modular integral $F_{\Delta}(z)$ of Duke, Imamoglu and Tóth [10] stated in the introduction.

6.2. Borcherds products. In this section we construct twisted Borcherds products of har-

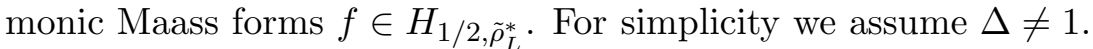

In order to generalize the Borcherds product to the full space $H_{1 / 2, \tilde{\rho}_{L}^{*}}$ we first recall the construction of certain weight 0 and weight 2 cocycles from [11, which will appear in the transformation rule of the Borcherds product.

Lemma 6.4. Let $n>0$ such that $N|\Delta| n$ is not a square, and let $\mathcal{A} \in \Gamma_{0}(N) \backslash L_{-|\Delta| n, r h}$. Then the function

$$
q_{M}^{\mathcal{A}}(z)=\sum_{\substack{X \in \mathcal{A} \\ a_{M X}<0<a_{X}}} \frac{1}{Q_{X}(z)}
$$

defines a weight 2 cocycle with values in the rational functions which are holomorphic on $\mathbb{H}$.

Proof. As in the proof of Proposition 6.1 we compute

$$
\sum_{\substack{X \in \mathcal{A} \\ a_{M X}<0<a_{X}}} \frac{1}{Q_{X}(z)}=\sum_{\substack{X \in \mathcal{A} \\ a>0}} \frac{\mathbf{1}_{X}(z) \operatorname{sgn}(a)}{Q_{X}(z)}-\left.\sum_{\substack{X \in \mathcal{A} \\ a>0}} \frac{\mathbf{1}_{X}(z) \operatorname{sgn}(a)}{Q_{X}(z)}\right|_{2} M
$$

for $z$ not lying on any geodesic $c_{X}$ with $X \in \mathcal{A}$. This easily implies that the map $M \mapsto q_{M}^{\mathcal{A}}$ is a weight 2 cocycle.

Next, we would like to construct a weight 0 cocycle $R_{M}^{\mathcal{A}}(z)$ with values in the holomorphic functions on $\mathbb{H}$ such that $\frac{\partial}{\partial z} R_{M}^{\mathcal{A}}(z)=q_{M}^{\mathcal{A}}(z)$. The following proposition gives such a construction for general cocycles with values in rational functions which are holomorphic on $\mathbb{H}$. 
Proposition 6.5 ([1], Theorem 2.1). Let $F(z)=\sum_{n \geq 0} a(n) e(n z)$ be a holomorphic modular integral of weight 2 for $\Gamma_{0}(N)$ with rational period functions $q_{M}=\left.F\right|_{2} M-F$. Assume that $a(n) \ll n^{\alpha}$ for some $\alpha>0$. For $M=\left(\begin{array}{ll}a & b \\ c & d\end{array}\right) \in \Gamma_{0}(N)$ with $c \neq 0$ we let

$$
\Lambda\left(s, \frac{a}{c}\right)=\left(\frac{2 \pi}{c}\right)^{-s} \Gamma(s) \sum_{n \geq 1} a(n) e\left(\frac{a n}{c}\right) n^{-s}
$$

and

$$
H\left(s, \frac{a}{c}\right)=\Lambda\left(s, \frac{a}{c}\right)+\int_{1}^{\infty} q_{M}(-d / c+i t / c) t^{1-s} d t+\frac{a(0)}{s}-\frac{a(0)}{2-s} .
$$

Then $H\left(s, \frac{a}{c}\right)$ is entire and satisfies the functional equation $H\left(s, \frac{a}{c}\right)=-H\left(2-s,-\frac{d}{c}\right)$. Further, for $c \neq 0$ we set

$$
R_{M}(z)=-\frac{i}{c} H\left(1, \frac{a}{c}\right)+\int_{-\frac{d}{c}+\frac{i}{c}}^{z} q_{M}(w) d w+a(0) \frac{a+d}{c},
$$

and for $M= \pm\left(\begin{array}{cc}1 & n \\ 0 & 1\end{array}\right)$ we let $R_{M}(z)=n a(0)$. Then $R_{M}(z)$ defines a weight 0 cocycle for $\Gamma_{0}(N)$ with values in the holomorphic functions on $\mathbb{H}$, and which satisfies $\frac{\partial}{\partial z} R_{M}(z)=q_{M}(z)$ for every $M \in \Gamma_{0}(N)$.

Proof. The proof is exactly the same as that of [11, Theorem 2.1, so we only give a sketch. By a standard computation we obtain for $c \neq 0$ the integral representation

$$
H\left(s, \frac{a}{c}\right)=-\int_{1}^{\infty}\left(F\left(z_{1 / t}\right)-a(0)\right) t^{1-s} d t+\int_{1}^{\infty}\left(F\left(M z_{t}\right)-a(0)\right) t^{s-1} d t
$$

where $z_{t}=-\frac{d}{c}+\frac{i}{c t}$. Since $z_{1 / t}=-\frac{d}{c}+\frac{i t}{c}$ and $M z_{t}=\frac{a}{c}+\frac{i t}{c}$, we see that $H\left(s, \frac{a}{c}\right)$ is entire and satisfies the claimed functional equation. Further, we let

$$
G(z)=a(0) z+\sum_{n \geq 1} \frac{a(n)}{2 \pi i n} e(n z)
$$

be a primitive of $F(z)$. By taking the limit $s \rightarrow 1$ in $H\left(s, \frac{a}{c}\right)$ we obtain after a short calculation

$$
R_{M}(z)=G(M z)-G(z)
$$

which is valid for all $M \in \Gamma_{0}(N)$ and defines a weight 0 cocycle with values in the holomorphic functions on $\mathbb{H}$, and $\frac{\partial}{\partial z} R_{M}(z)=q_{M}(z)$.

Lemma 6.6. Let $q_{M}^{\mathcal{A}}$ be the weight 2 cocycle associated to $\mathcal{A} \in \Gamma_{0}(N) \backslash L_{-|\Delta| n, r h}$ as above. For $X \in \mathcal{A}$ let $w_{X}>w_{X}^{\prime}$ denote the two real endpoints of the geodesic $c_{X}$. Let $F(z)=$ $\sum_{n \geq 0} a(n) q^{n}$ be a modular integral for $q_{M}^{\mathcal{A}}$ with $a(n) \ll n^{\alpha}$ for some $\alpha>0$ and let $M=$ $\left(\begin{array}{ll}a & b \\ c & d\end{array}\right) \in \Gamma_{0}(N)$. Further, for $c \neq 0$ let

$$
L_{F}\left(s, \frac{a}{c}\right)=\sum_{n \geq 1} a(n) e\left(\frac{a n}{c}\right) n^{-s} .
$$

and

$$
R_{M}^{\mathcal{A}}(z)=\frac{1}{\sqrt{4 N|\Delta| n}} \sum_{\substack{X \in \mathcal{A} \\ a_{M X}<0<a_{X}}}\left(\log \left(z-w_{X}\right)-\log \left(z-w_{X}^{\prime}\right)\right)+\frac{1}{2 \pi i} L_{F}\left(1, \frac{a}{c}\right)+a(0) \frac{a+d}{c},
$$

and for $M= \pm\left(\begin{array}{ll}1 & n \\ 0 & 1\end{array}\right)$ we let $R_{M}^{\mathcal{A}}(z)=n a(0)$. Then $R_{M}^{\mathcal{A}}(z)$ is a weight 0 cocycle with values in the holomorphic functions on $\mathbb{H}$ which satisfies $\frac{\partial}{\partial z} R_{M}^{\mathcal{A}}(z)=q_{M}^{\mathcal{A}}(z)$. 
Proof. Note that

$$
q_{M}^{\mathcal{A}}(z)=\frac{1}{\sqrt{4 N|\Delta| n}} \sum_{\substack{X \in \mathcal{A} \\ a_{M X}<0<a_{X}}}\left(\frac{1}{z-w_{X}}-\frac{1}{z-w_{X}^{\prime}}\right) .
$$

Thus if we choose

$$
\frac{1}{\sqrt{4 N|\Delta| n}} \sum_{\substack{X \in \mathcal{A} \\ a_{M X}<0<a_{X}}}\left(\log \left(z-w_{X}\right)-\log \left(z-w_{X}^{\prime}\right)\right)
$$

as a primitive for $q_{M}^{\mathcal{A}}(z)$, the formula for $R_{M}^{\mathcal{A}}(z)$ follows from Proposition 6.5,

Example 6.7. Let $N=1, \Delta>1$, and $M=S=\left(\begin{array}{cc}0 & -1 \\ 1 & 0\end{array}\right)$. We have

$$
q_{S}^{\mathcal{A}}(z)=\sum_{\substack{X \in \mathcal{A} \\ c<0<a}} \frac{1}{Q_{X}(z)}
$$

It easily follows from the definition and the functional equation of $H(s, 0)$ given in Proposition 6.5 that

$$
L_{F}(1,0)=-\frac{2 \pi i}{\sqrt{4 \Delta n}} \sum_{\substack{X \in \mathcal{A} \\ c<0<a}}\left(\log \left(i-w_{X}\right)-\log \left(i-w_{X}^{\prime}\right)\right)
$$

independently of the modular integral $F$ for $q^{\mathcal{A}}$. In particular, we obtain

$$
R_{S}^{\mathcal{A}}(z)=\frac{1}{\sqrt{4 \Delta n}} \sum_{\substack{X \in \mathcal{A} \\ c<0<a}}\left(\log \left(\frac{z-w_{X}}{i-w_{X}}\right)-\log \left(\frac{z-w_{X}^{\prime}}{i-w_{X}^{\prime}}\right)\right) .
$$

We can now state the transformation behaviour of the Borcherds product associated to $f \in H_{1 / 2, \tilde{\rho}_{L}^{*}}$.

Theorem 6.8. Let $\Delta \neq 1$ be a fundamental discriminant. Let $f \in H_{1 / 2, \tilde{\rho}_{L}^{*}}$ and suppose that $c_{f}^{+}\left(|\Delta| m^{2} / 4 N, r m\right) \in \mathbb{R}$ for all $m \in \mathbb{Z}, m>0$. Further, assume that $c_{f}^{+}(n, h)=0$ for all $n<0, h \in L^{\prime} / L$, and that $c_{f}^{-}\left(|\Delta| m^{2} / 4 N, r m\right)=0$ for all $m \in \mathbb{Z}, m>0$. Then the infinite product

$$
\Psi_{\Delta, r}(f, z)=e\left(\frac{\sqrt{|\Delta| N}}{4 \pi} L_{\Delta}(1) c_{f}^{-}(0,0) z\right) \prod_{m=1}^{\infty} \prod_{b(\Delta)}[1-e(m z+b / \Delta)]^{\left(\frac{\Delta}{b}\right) c_{f}^{+}\left(|\Delta| m^{2} / 4 N, r m\right)}
$$

converges to a holomorphic function on $\mathbb{H}$ transforming as

$$
\Psi_{\Delta, r}(f, M z)=\chi(M) \mu_{\Delta, r}(f, M, z) \Psi_{\Delta, r}(f, z)
$$

for all $M \in \Gamma_{0}(N)$, where $\chi$ is a character of $\Gamma_{0}(N)$ and

$$
\mu_{\Delta, r}(f, M, z)=\prod_{h \in L^{\prime} / L} \prod_{n>0} \prod_{\mathcal{A} \in \Gamma_{0}(N) \backslash L_{-|\Delta| n, r h}} e\left(-\frac{\sqrt{|\Delta| N}}{\pi} c_{f}^{-}(n, h) \chi_{\Delta}(\mathcal{A}) R_{M}^{\mathcal{A}}(z)\right),
$$

where $R_{M}^{\mathcal{A}}(z)$ is the weight 0 cocycle with $\frac{\partial}{\partial z} R_{M}^{\mathcal{A}}(z)=q_{M}^{\mathcal{A}}(z)$. Further, its logarithmic derivative is given by

$$
\frac{\partial}{\partial z} \log \left(\Psi_{\Delta, r}(f, z)\right)=-2 \pi i \sqrt{|\Delta| N} F_{\Delta, r}(f, z),
$$

where $F_{\Delta, r}(f, z)$ is the modular integral defined in Proposition 6.1. 
Proof. Using Proposition 6.1 we see after a short calculation that the logarithmic derivatives of $\Psi_{\Delta, r}(f, M z)$ and $\mu_{\Delta, r}(f, M, z) \Psi_{\Delta, r}(f, z)$ agree. Further, both functions are holomorphic and non-vanishing on $\mathbb{H}$. Hence they are constant multiples of each other. This proves the transformation behaviour.

The fact that $R_{M}^{\mathcal{A}}(z)$ is a weight 0 cocycle together with the transformation formula of the Borcherds product implies that $\chi$ is a character of $\Gamma_{0}(N)$.

Example 6.9. Let $\Delta>1$, and let $f \in H_{1 / 2, \rho_{L}^{*}}$ be the Bruinier-Funke-Imamoglu lift of a harmonic Maass form $F \in H_{0}^{+}\left(\Gamma_{0}(N)\right)$ with vanishing constant coefficients $a_{\ell}^{+}(0)$ at all cusps as in Example 6.3. Its Borcherds lift is given by

$$
\begin{aligned}
\Psi_{\Delta, r}\left(\frac{\pi}{\sqrt{N}} f, z\right)= & \prod_{m=1}^{\infty} \prod_{b(\Delta)}[1-e(m z+b / \Delta)]\left(\frac{\Delta}{b}\right) \operatorname{tr}_{F}\left(-\Delta m^{2} / 4 N, r m\right) \\
& \times e\left(-\frac{\sqrt{\Delta}}{2} L_{\Delta}(1) \operatorname{tr}_{F}(0,0) z\right) .
\end{aligned}
$$

For $N=1$ and $F=J=j-744$ (with $\operatorname{tr}_{J}(0,0)=4$ and $\operatorname{tr}_{J}^{c}(-1 / 4,1)=2$ ) we obtain the theorem in the introduction. Note that the relations $S^{4}=1,(S T)^{6}=1$ and $\chi(T)=1$ imply that $\chi=1$ for $N=1$.

\section{REFERENCES}

[1] Milton Abramowitz and Irene A. Stegun. Handbook of mathematical functions with formulas, graphs, and mathematical tables, volume 55 of National Bureau of Standards Applied Mathematics Series. 1964.

[2] Richard E. Borcherds. Automorphic forms on $O_{s+2,2}(r)$ and infinite products. Invent. Math., 120:161-213, 1995.

[3] Richard E. Borcherds. Automorphic forms with singularities on Grassmannians. Invent. Math., 132(3):491-562, 1998.

[4] Jan H. Bruinier. Borcherds products on $O(2, l)$ and Chern classes of Heegner divisors, volume 1780 of Lecture Notes in Mathematics. Springer-Verlag, Berlin, 2002.

[5] Jan H. Bruinier and Jens Funke. On two geometric theta lifts. Duke Math. J., 125(1):45-90, 2004.

[6] Jan H. Bruinier and Jens Funke. Traces of CM values of modular functions. J. Reine Angew. Math., 594:1-33, 2006.

[7] Jan H. Bruinier, Jens Funke, and Özlem Imamoglu. Regularized theta liftings and periods of modular functions. J. reine angew. Math., 2015(703):43-93, 2015.

[8] Jan H. Bruinier and Ken Ono. Heegner divisors, L-functions and harmonic weak Maass forms. Ann. of Math. (2), 172(3):2135-2181, 2010.

[9] William Duke, Özlem Imamoglu, and Árpad Tóth. Rational period functions and cycle integrals. Abh. Math. Semin. Univ. Hambg., 80(2):255-264, 2010.

[10] William Duke, Özlem Imamoglu, and Árpad Tóth. Cycle integrals of the $j$-function and mock modular forms. Ann. Math., 173(2):947-981, 2011.

[11] William Duke, Özlem Imamoglu, and Árpad Tóth. Linking numbers and modular cocycles. Duke Math. J., 166(6):1179-1210, 2017.

[12] Martin Eichler and Don Zagier. The theory of Jacobi forms, volume 55 of Progress in Mathematics. Birkhäuser Boston Inc., Boston, MA, 1985.

[13] A. Erdélyi, W. Magnus, F. Oberhettinger, and F. G. Tricomi. Tables of integral transforms. Vol. I. McGraw-Hill Book Company, Inc., New York-Toronto-London, 1954. Based, in part, on notes left by Harry Bateman.

[14] Marvin I. Knopp. Some new results on the Eichler cohomology of automorphic forms. Bull. Amer. Math. Soc., 80(4):607-632, 1974. 
[15] Don Zagier. Eisenstein series and the Riemann zeta-function. Automorphic Forms, Representation Theory and Arithmetic: Papers presented at the Bombay Colloquium 1979. Springer-Verlag, Berlin-HeidelbergNew York, pages 275-301, 1981.

[16] Don Zagier. Traces of singular moduli. In Motives, polylogarithms and Hodge theory, Part I (Irvine, CA, 1998), volume 3 of Int. Press Lect. Ser., pages 211-244. Int. Press, Somerville, MA, 2002.

Mathematical Institute, University of Cologne, Weyertal 86-90, D-50931 Cologne, Germany E-mail address: mschwage@math.uni-koeln.de 